\title{
Behavior of the solutions to third order linear autonomous delay differential equations
}

\author{
Ali Fuat Yenicerioglu \\ Department of Mathematics, The Faculty of Education, Kocaeli University, Kocaeli, Turkey \\ Received: 1 May 2017, Accepted: 9 November 2017 \\ Published online: 17 May 2018.
}

\begin{abstract}
A wide class of third order linear autonomous delay differential equations with distributed type delays is considered. An asymptotic result, a useful exponential estimate of the solutions, a stability criterion, and a result on the bahavior of the solutions are established.
\end{abstract}

Keywords: Delay differential equation, characteristic equation, roots, asymptotic behavior, solution, stability,

\section{Introduction and preliminaries}

The theory of delay differential equations is important both theoretical and practical interest. For the basic theory of delay differential equations, the reader is referred to the books by Bellman and Cooke [1], Driver [4], El'sgol'ts and Norkin [5], Hale and Verduyn Lunel [6], Kolmanovski and Myshkis [7] and Lakshmikantham, Wen and Zhang [8].

In this paper, we deal with the stability of the trivial solution for a third order linear autonomous delay differential equation with constant delay. An asymptotic result for the solutions is obtained. Also, an estimate of the solutions and a stability criterion for the trivial solution are established. The sufficient conditions for the stability and the asymptotic stability of the trivial solution and some examples are given. Moreover, a result on the behavior of the solutions is given. Our results are derived by the use of real roots (with an appropriate property) of the corresponding (in a sense) characteristic equations. The very interesting asymptotic and stability results were given by Philos and Purnaras [9-10]. The techniques applied in $[11,12]$ are originated in a combination of the methods used in $[9,10]$.

Yeniçerioğlu [12] obtained some results on the qualitative behavior of the solutions of a second order linear autonomous delay differential equation with a single delay. The main idea in [12] is that of transforming the second order delay differential equation into a first order delay differential equation, by the use of a real root of the corresponding characteristic equation. The same idea will be used in this paper to obtain some general results.

Recently, Cahlon and Schmidt et al. [2] have established the stability criteria for a third order delay differential equation. This equation is obtained the stability of third order delay differential equation using Pontryagin's theory for quasi-polynomials. However, we study the stability of the some problem using the method of characteristic roots.

Let us consider initial value problem for third order delay differential equation

$$
y^{\prime \prime \prime}=p_{1} y^{\prime \prime}(t)+p_{2} y^{\prime \prime}(t-\tau)+q_{1} y^{\prime}(t)+q_{2} y^{\prime}(t-\tau)+v_{1} y(t)+v_{2} y(t-\tau), t \geq 0
$$

where $p_{1}, p_{2}, q_{1}, q_{2}, v_{1}, v_{2}$ are real numbers, $\tau$ is a positive real number. In a previous paper [3], we considered Eq. (1) 
with $q_{2}=0$ and $v_{1}=0$ which arose from a robotic model with damping and delay. There are no practical stability criteria of the zero solution of (1).

By a solution of the delay differential equation (1), we mean a twice continuously differentiable real-valued function $y$ defined on the interval $[-\tau, \infty)$, which is thrice continuously differentiable on $[0, \infty)$ and satisfies (1) for all $t \geq 0$. Together with the delay differential equation (1), it is customary to specify an initial condition of the form

$$
y(t)=\phi(t), \text { for } \quad-\tau \leq t \leq 0,
$$

where the initial function $\phi(t)$ is a given twice continuously differentiable real-valued function on the initial interval $[-\tau, 0]$.

Equations (1) and (2) constitute an initial value problem (IVP, for short). It is known that, ( see, for example, Driver [4] ) for any given initial function $\phi$, there exists a unique solution of the initial value problem (1) and (2) or, more briefly, the solution of the IVP (1) and (2).

Along with the delay differential equation (1), we associate the equation

$$
\lambda^{3}=\lambda^{2} p_{1}+\lambda q_{1}+v_{1}+e^{-\lambda \tau}\left(\lambda^{2} p_{2}+\lambda q_{2}+v_{2}\right)
$$

which will be called the characteristic equation of (1). Eq. (3) is obtained from (1) by looking for solutions of the form $y(t)=e^{\lambda t}$ for $t \geq-\tau$.

For a given real root $\lambda_{0}$ of the characteristic equation (3), we consider the (second order) delay differential equation

$$
\begin{aligned}
z^{\prime \prime}(t)= & \left(p_{1}-3 \lambda_{0}\right) z^{\prime}(t)+e^{-\lambda_{0} \tau} p_{2} z^{\prime}(t-\tau)+\left(q_{1}+2 p_{1} \lambda_{0}-3 \lambda_{0}^{2}\right) z(t) \\
& +e^{-\lambda_{0} \tau}\left(q_{2}+2 p_{2} \lambda_{0}\right) z(t-\tau)-e^{-\lambda_{0} \tau}\left(p_{2} \lambda_{0}^{2}+q_{2} \lambda_{0}+v_{2}\right) \int_{t-\tau}^{t} z(s) d s .
\end{aligned}
$$

A solution of the delay differential equation (3) is a continuous real-valued function $z$ defined on the interval $[-\tau, \infty)$, which is twice continuously differentiable on $[0, \infty)$ and satisfies (3) for all $t \geq 0$.

With the delay differential equation (3), we associate the equation

$$
\begin{aligned}
\delta^{2}= & \left(p_{1}-3 \lambda_{0}\right) \delta+q_{1}+2 p_{1} \lambda_{0}-3 \lambda_{0}^{2}+\left(p_{2} \delta+q_{2}+2 p_{2} \lambda_{0}\right) e^{-\left(\lambda_{0}+\delta\right) \tau} \\
& -e^{-\lambda_{0} \tau}\left(p_{2} \lambda_{0}^{2}+q_{2} \lambda_{0}+v_{2}\right) \delta^{-1}\left(1-e^{-\delta \tau}\right)
\end{aligned}
$$

which is said to be the characteristic equation of (3). This equation is obtained from (3) by seeking solutions of the form $z(t)=e^{\delta t}$ for $t \geq-\tau$.

For our convenience, we introduce some notations. For a given real root $\lambda_{0}$ of the characteristic equation (3), we set

$$
\beta_{\lambda_{0}}=e^{-\lambda_{0} \tau}\left(\left(p_{2} \lambda_{0}^{2}+q_{2} \lambda_{0}+v_{2}\right) \tau-q_{2}-2 p_{2} \lambda_{0}\right)-q_{1}-2 p_{1} \lambda_{0}+3 \lambda_{0}^{2}
$$

and, also, we define

$$
\begin{aligned}
L\left(\lambda_{0} ; \phi\right)= & \phi^{\prime \prime}(0)+\left(\lambda_{0}-p_{1}\right) \phi^{\prime}(0)-p_{2} \phi^{\prime}(-\tau)+\left(\lambda_{0}^{2}-p_{1} \lambda_{0}-q_{1}\right) \phi(0) \\
& -\left(p_{2} \lambda_{0}+q_{2}\right) \phi(-\tau)+e^{-\lambda_{0} \tau}\left(p_{2} \lambda_{0}^{2}+q_{2} \lambda_{0}+v_{2}\right) \int_{-\tau}^{0} e^{-\lambda_{0} s} \phi(s) d s
\end{aligned}
$$


in addition, provided that $\beta_{\lambda_{0}} \neq 0$, we define

$$
\Phi_{1}\left(\lambda_{0} ; \phi\right)(t)=\phi(t) e^{-\lambda_{0} t}-\frac{L\left(\lambda_{0} ; \phi\right)}{\beta_{\lambda_{0}}} \text { for } \quad-\tau \leq t \leq 0 .
$$

We will now give a proposition, which plays a crucial role in obtaining our main results.

Proposition 1. Let $\lambda_{0}$ be real root of the characteristic equation (3), and let $\beta_{\lambda_{0}}$ and $L\left(\lambda_{0} ; \phi\right)$ be defined by (6) and (7), respectively. Suppose that $\beta_{\lambda_{0}} \neq 0$, and define $\Phi_{1}\left(\lambda_{0} ; \phi\right)$ by (8). Then a continuous real-valued function y defined on the interval $[-\tau, \infty)$ is the solution of the IVP (1) and (2)if and only if the function $z$ defined by

$$
z(t)=y(t) e^{-\lambda_{0} t}-\frac{L\left(\lambda_{0} ; \phi\right)}{\beta_{\lambda_{0}}} \text { for } t \geq-\tau
$$

is the solution of the delay differential equation (4) which satisfies the initial condition

$$
z(t)=\Phi_{1}\left(\lambda_{0} ; \phi\right)(t) \text { for } \quad-\tau \leq t \leq 0 .
$$

Proof. Let $y$ be the solution of the IVP (1) and (2). Define

$$
x(t)=e^{-\lambda_{0} t} y(t) \text { for } t \in[-\tau, \infty),
$$

where $\lambda_{0}$ is a real root of the characteristic equation (3). Then, for every $t \geq 0$, we have

$$
\begin{aligned}
& {\left[x^{\prime \prime}(t)+\left(3 \lambda_{0}-p_{1}\right) x^{\prime}(t)-e^{-\lambda_{0} \tau} p_{2} x^{\prime}(t-\tau)+\left(3 \lambda_{0}^{2}-2 p_{1} \lambda_{0}-q_{1}\right) x(t)-e^{-\lambda_{0} \tau}\left(2 p_{2} \lambda_{0}+q_{2}\right) x(t-\tau)\right]^{\prime}} \\
& =\left(p_{1} \lambda_{0}^{2}+q_{1} \lambda_{0}+v_{1}-\lambda_{0}^{3}\right) x(t)+e^{-\lambda_{0} \tau}\left(p_{2} \lambda_{0}^{2}+q_{2} \lambda_{0}+v_{2}\right) x(t-\tau) .
\end{aligned}
$$

Moreover, the initial condition (2) can be equivalently written

$$
x(t)=e^{-\lambda_{0} t} \phi(t) \text { for } t \in[-\tau, 0] .
$$

Furthermore, by using (3) and taking into account (13), we can verify that (12) is equivalent to

$$
\begin{gathered}
x^{\prime \prime}(t)+\left(3 \lambda_{0}-p_{1}\right) x^{\prime}(t)-e^{-\lambda_{0} \tau} p_{2} x^{\prime}(t-\tau)+\left(3 \lambda_{0}^{2}-2 p_{1} \lambda_{0}-q_{1}\right) x(t)-e^{-\lambda_{0} \tau}\left(2 p_{2} \lambda_{0}+q_{2}\right) x(t-\tau) \\
=\left(p_{1} \lambda_{0}^{2}+q_{1} \lambda_{0}+v_{1}-\lambda_{0}^{3}\right) \int_{0}^{t} x(s) d s+e^{-\lambda_{0} \tau}\left(p_{2} \lambda_{0}^{2}+q_{2} \lambda_{0}+v_{2}\right) \int_{0}^{t} x(s-\tau) d s+x^{\prime \prime}(0)+\left(3 \lambda_{0}-p_{1}\right) x^{\prime}(0) \\
-e^{-\lambda_{0} \tau} p_{2} x^{\prime}(-\tau)+\left(3 \lambda_{0}^{2}-2 p_{1} \lambda_{0}-q_{1}\right) x(0)-e^{-\lambda_{0} \tau}\left(2 p_{2} \lambda_{0}+q_{2}\right) x(-\tau) \\
x^{\prime \prime}(t)=\left(p_{1}-3 \lambda_{0}\right) x^{\prime}(t)+e^{-\lambda_{0} \tau} p_{2} x^{\prime}(t-\tau)+\left(q_{1}+2 p_{1} \lambda_{0}-3 \lambda_{0}^{2}\right) x(t)+e^{-\lambda_{0} \tau}\left(q_{2}+2 p_{2} \lambda_{0}\right) x(t-\tau) \\
+\left(p_{1} \lambda_{0}^{2}+q_{1} \lambda_{0}+v_{1}-\lambda_{0}^{3}\right) \int_{0}^{t} x(s) d s+e^{-\lambda_{0} \tau}\left(p_{2} \lambda_{0}^{2}+q_{2} \lambda_{0}+v_{2}\right) \int_{-\tau}^{t-\tau} x(s) d s \\
+\phi^{\prime \prime}(0)-2 \lambda_{0} \phi^{\prime}(0)+\lambda_{0}^{2} \phi(0)+\left(3 \lambda_{0}-p_{1}\right)\left(\phi^{\prime}(0)-\lambda_{0} \phi(0)\right)-p_{2}\left(\phi^{\prime}(-\tau)-\lambda_{0} \phi(-\tau)\right) \\
+\left(3 \lambda_{0}^{2}-2 p_{1} \lambda_{0}-q_{1}\right) \phi(0)-\left(2 p_{2} \lambda_{0}+q_{2}\right) \phi(-\tau), \\
x^{\prime \prime}(t)=\left(p_{1}-3 \lambda_{0}\right) x^{\prime}(t)+e^{-\lambda_{0} \tau} p_{2} x^{\prime}(t-\tau)+\left(q_{1}+2 p_{1} \lambda_{0}-3 \lambda_{0}^{2}\right) x(t) \\
+e^{-\lambda_{0} \tau}\left(q_{2}+2 p_{2} \lambda_{0}\right) x(t-\tau)+\left(p_{1} \lambda_{0}^{2}+q_{1} \lambda_{0}+v_{1}-\lambda_{0}^{3}\right) \int_{0}^{t} x(s) d s \\
+e^{-\lambda_{0} \tau}\left(p_{2} \lambda_{0}^{2}+q_{2} \lambda_{0}+v_{2}\right) \int_{0}^{t-\tau} x(s) d s+L\left(\lambda_{0} ; \phi\right),
\end{gathered}
$$




$$
\begin{gathered}
x^{\prime \prime}(t)=\left(p_{1}-3 \lambda_{0}\right) x^{\prime}(t)+e^{-\lambda_{0} \tau} p_{2} x^{\prime}(t-\tau)+\left(q_{1}+2 p_{1} \lambda_{0}-3 \lambda_{0}^{2}\right) x(t) \\
+e^{-\lambda_{0} \tau}\left(q_{2}+2 p_{2} \lambda_{0}\right) x(t-\tau)-e^{-\lambda_{0} \tau}\left(p_{2} \lambda_{0}^{2}+q_{2} \lambda_{0}+v_{2}\right) \int_{0}^{t} x(s) d s \\
+e^{-\lambda_{0} \tau}\left(p_{2} \lambda_{0}^{2}+q_{2} \lambda_{0}+v_{2}\right) \int_{0}^{t-\tau} x(s) d s+L\left(\lambda_{0} ; \phi\right), \\
x^{\prime \prime}(t)=\left(p_{1}-3 \lambda_{0}\right) x^{\prime}(t)+e^{-\lambda_{0} \tau} p_{2} x^{\prime}(t-\tau)+\left(q_{1}+2 p_{1} \lambda_{0}-3 \lambda_{0}^{2}\right) x(t) \\
+e^{-\lambda_{0} \tau}\left(q_{2}+2 p_{2} \lambda_{0}\right) x(t-\tau)-e^{-\lambda_{0} \tau}\left(p_{2} \lambda_{0}^{2}+q_{2} \lambda_{0}+v_{2}\right) \int_{t-\tau}^{t} x(s) d s+L\left(\lambda_{0} ; \phi\right),
\end{gathered}
$$

where $L\left(\lambda_{0} ; \phi\right)$ was given in (7). Now, we take into account the assumption $\beta_{\lambda_{0}} \neq 0$ and we define

$$
z(t)=x(t)-\frac{L\left(\lambda_{0} ; \phi\right)}{\beta_{\lambda_{0}}} \text { for } t \geq-r
$$

Then, because of definition of $\beta_{\lambda_{0}}$ by (6), it is a matter of elementary calculations to show that $x$ satisfies (14) for $t \geq 0$ if and only if $z$ satisfies (4) for $t \geq 0$, i.e., if and only if $z$ is a solution of the delay differential equation (4). Moreover, we see that the initial condition (13) is equivalently written as follows

$$
z(t)=e^{-\lambda_{0} t} \phi(t)-\frac{L\left(\lambda_{0} ; \phi\right)}{\beta_{\lambda_{0}}} \text { for } \quad-\tau \leq t \leq 0 .
$$

We have thus proved that $y$ is the solution of the IVP (1) and (2) if and only if $z$ is the solution of the delay differential equation (4) which satisfies the initial condition (16). By (11), we see that (15) coincides with (9). Also, by taking into account the definition of $\Phi_{1}\left(\lambda_{0} ; \phi\right)$ by (8), we observe that (16) coincides with the initial condition (10). The proof of the proposition 1 is completed.

For a given real roots $\lambda_{0}, \delta_{0}$ of the characteristic equations (3) and (5), respectively, we consider the (first order) delay differential equation

$$
\begin{aligned}
w^{\prime}(t)= & \left(p_{1}-3 \lambda_{0}-2 \delta_{0}\right) w(t)+e^{-\left(\lambda_{0}+\delta_{0}\right) \tau} p_{2} w(t-\tau)-e^{-\left(\lambda_{0}+\delta_{0}\right) \tau}\left(p_{2} \delta_{0}+q_{2}+2 p_{2} \lambda_{0}\right) \int_{t-\tau}^{t} w(s) d s \\
& +e^{-\lambda_{0} \tau}\left(p_{2} \lambda_{0}^{2}+q_{2} \lambda_{0}+v_{2}\right) \int_{0}^{\tau} e^{-\delta_{0} s}\left\{\int_{t-s}^{t} w(u) d u\right\} d s .
\end{aligned}
$$

By a solution of the delay (17), we mean a continuous real-valued function $w$ defined on the interval $[-\tau, \infty)$, which is continuously differentiable on $[0, \infty)$ and satisfies (17) for all $t \geq 0$. The characteristic equation of the delay differential equation (17) is

$$
\begin{aligned}
\gamma= & p_{1}-3 \lambda_{0}-2 \delta_{0}+p_{2} e^{-\left(\lambda_{0}+\delta_{0}+\gamma\right) \tau}-e^{-\left(\lambda_{0}+\delta_{0}\right) \tau}\left(p_{2} \delta_{0}+q_{2}+2 p_{2} \lambda_{0}\right) \int_{0}^{\tau} e^{-\gamma s} d s \\
& +e^{-\lambda_{0} \tau}\left(p_{2} \lambda_{0}^{2}+q_{2} \lambda_{0}+v_{2}\right) \int_{0}^{\tau} e^{-\delta_{0} s}\left(\int_{0}^{s} e^{-\gamma u} d u\right) d s
\end{aligned}
$$

or

$$
\begin{aligned}
\gamma= & p_{1}-3 \lambda_{0}-2 \delta_{0}+p_{2} e^{-\left(\lambda_{0}+\delta_{0}+\gamma\right) \tau}-e^{-\left(\lambda_{0}+\delta_{0}\right) \tau}\left(p_{2} \delta_{0}+q_{2}+2 p_{2} \lambda_{0}\right) \gamma^{-1}\left(1-e^{-\gamma \tau}\right) \\
& +e^{-\lambda_{0} \tau}\left(p_{2} \lambda_{0}^{2}+q_{2} \lambda_{0}+v_{2}\right) \gamma^{-1}\left\{\delta_{0}^{-1}\left(1-e^{-\delta_{0} \tau}\right)-\left(\delta_{0}+\gamma\right)^{-1}\left(1-e^{-\left(\delta_{0}+\gamma\right) \tau}\right)\right\}
\end{aligned}
$$

The last equation is obtained from (17) by seeking solutions of the form $w(t)=e^{\gamma t}$ for $t \geq-\tau$.

For our convenience, we introduce some notations. For a given real root $\lambda_{0}$ of the characteristic equation (3) and a given 
real root $\delta_{0}$ of the characteristic equation (5), we set

$$
\eta_{\lambda_{0}, \delta_{0}}=e^{-\left(\lambda_{0}+\delta_{0}\right) \tau}\left\{\left(p_{2} \delta_{0}+q_{2}+2 p_{2} \lambda_{0}\right) \tau-p_{2}\right\}-p_{1}+3 \lambda_{0}+2 \delta_{0}-e^{-\lambda_{0} \tau}\left(p_{2} \lambda_{0}^{2}+q_{2} \lambda_{0}+v_{2}\right) \int_{0}^{\tau} s e^{-\delta_{0} s} d s
$$

and let $\Phi_{1}\left(\lambda_{0} ; \phi\right)$ be defined by (8). Also, we define

$$
\begin{aligned}
R\left(\lambda_{0}, \delta_{0} ; \phi\right)= & \left(\Phi_{1}\left(\lambda_{0} ; \phi\right)\right)^{\prime}(0)-\delta_{0} \Phi_{1}\left(\lambda_{0} ; \phi\right)(0)-\left(p_{1}-3 \lambda_{0}-2 \delta_{0}\right) \Phi_{1}\left(\lambda_{0} ; \phi\right)(0) \\
& -e^{-\lambda_{0} \tau} p_{2} \Phi_{1}\left(\lambda_{0} ; \phi\right)(-\tau)+e^{-\left(\lambda_{0}+\delta_{0}\right) \tau}\left(p_{2} \delta_{0}+q_{2}+2 p_{2} \lambda_{0}\right) \int_{-\tau}^{0} e^{-\delta_{0} s} \Phi_{1}\left(\lambda_{0} ; \phi\right)(s) d s \\
& -e^{-\lambda_{0} \tau}\left(p_{2} \lambda_{0}^{2}+q_{2} \lambda_{0}+v_{2}\right) \int_{0}^{\tau} e^{-\delta_{0} s}\left\{\int_{-s}^{0} e^{-\delta_{0} u} \Phi_{1}\left(\lambda_{0} ; \phi\right)(u) d u\right\} d s,
\end{aligned}
$$

where $\left(\Phi_{1}\left(\lambda_{0} ; \phi\right)\right)^{\prime}$ is derivative of $\Phi_{1}\left(\lambda_{0} ; \phi\right)$; in addition, provided that $\eta_{\lambda_{0}, \delta_{0}} \neq 0$, we define

$$
\Phi_{2}\left(\lambda_{0}, \delta_{0} ; \phi\right)(t)=e^{-\delta_{0} t} \Phi_{1}\left(\lambda_{0} ; \phi\right)(t)-\frac{R\left(\lambda_{0}, \delta_{0} ; \phi\right)}{\eta_{\lambda_{0}, \delta_{0}}} \text { for } t \in[-\tau, 0]
$$

We will now give a proposition, which plays a crucial role in obtaining our main results.

Proposition 2. Let $\delta_{0}$ be real root of the characteristic equation (5), and let $\eta_{\lambda_{0}, \delta_{0}}$ and $R\left(\lambda_{0}, \delta_{0} ; \phi\right)$ be defined by (19) and (20), respectively. Suppose that $\eta_{\lambda_{0}, \delta_{0}} \neq 0$ and define $\Phi_{2}\left(\lambda_{0}, \delta_{0} ; \phi\right)$ by (21). Then a continuous real-valued function $z$ defined on the interval $[-\tau, \infty)$ is the solution of the delay differential equation (4) which satisfies the initial condition (10) if and only if the function $w$ defined by

$$
w(t)=e^{-\delta_{0} t} z(t)-\frac{R\left(\lambda_{0}, \delta_{0} ; \phi\right)}{\eta_{\lambda_{0}, \delta_{0}}} \text { for } t \geq-\tau
$$

is the solution of the delay differential equation (17) which satisfies the initial condition

$$
w(t)=\Phi_{2}\left(\lambda_{0}, \delta_{0} ; \phi\right)(t) \text { for } \quad-\tau \leq t \leq 0 .
$$

Proof. Let now $z$ be the solution of (4) and (10) and $\delta_{0}$ be a real root of the characteristic equation (5). Define

$$
v(t)=e^{-\delta_{0} t} z(t) \text { for all } t \in[-\tau, \infty) .
$$

Then, for every $t \geq 0$, we have

$$
\begin{aligned}
v^{\prime \prime}(t)= & \left(p_{1}-3 \lambda_{0}-2 \delta_{0}\right) v^{\prime}(t)+e^{-\left(\lambda_{0}+\delta_{0}\right) \tau} p_{2} v^{\prime}(t-\tau)+\left(\delta_{0} p_{1}-3 \lambda_{0} \delta_{0}+q_{1}+2 p_{1} \lambda_{0}-3 \lambda_{0}^{2}-\delta_{0}^{2}\right) v(t) \\
& +e^{-\left(\lambda_{0}+\delta_{0}\right) \tau}\left(\delta_{0} p_{2}+q_{2}+2 p_{2} \lambda_{0}\right) v(t-\tau)-e^{-\lambda_{0} \tau}\left(p_{2} \lambda_{0}^{2}+q_{2} \lambda_{0}+v_{2}\right) \int_{0}^{\tau} e^{-\delta_{0} s} v(t-s) d s .
\end{aligned}
$$

Moreover, the initial condition (16) can be equivalently written

$$
v(t)=e^{-\delta_{0} t} \Phi_{1}\left(\lambda_{0} ; \phi\right)(t) \text { for } t \in[-\tau, 0],
$$

where $\Phi_{1}$ was given in (8). Furthermore, by using (5) and taking into account (26), we can verify that (25) is equivalent to

$$
\begin{aligned}
v^{\prime}(t)= & v^{\prime}(0)+\left(p_{1}-3 \lambda_{0}-2 \delta_{0}\right) v(t)-\left(p_{1}-3 \lambda_{0}-2 \delta_{0}\right) v(0)+e^{-\left(\lambda_{0}+\delta_{0}\right) \tau} p_{2} v(t-\tau)-e^{-\left(\lambda_{0}+\delta_{0}\right) \tau} p_{2} v(-\tau) \\
& +\left(\delta_{0} p_{1}-3 \lambda_{0} \delta_{0}+q_{1}+2 p_{1} \lambda_{0}-3 \lambda_{0}^{2}-\delta_{0}^{2}\right) \int_{0}^{t} v(s) d s+e^{-\left(\lambda_{0}+\delta_{0}\right) \tau}\left(\delta_{0} p_{2}+q_{2}+2 p_{2} \lambda_{0}\right) \int_{0}^{t} v(s-\tau) d s \\
& -e^{-\lambda_{0} \tau}\left(p_{2} \lambda_{0}^{2}+q_{2} \lambda_{0}+v_{2}\right) \int_{0}^{\tau} e^{-\delta_{0} s}\left\{\int_{0}^{t} v(u-s) d u\right\} d s,
\end{aligned}
$$




$$
\begin{aligned}
v^{\prime}(t)= & \left(\Phi_{1}\left(\lambda_{0} ; \phi\right)\right)^{\prime}(0)-\delta_{0} \Phi_{1}\left(\lambda_{0} ; \phi\right)(0)+\left(p_{1}-3 \lambda_{0}-2 \delta_{0}\right) v(t)-\left(p_{1}-3 \lambda_{0}-2 \delta_{0}\right) \Phi_{1}\left(\lambda_{0} ; \phi\right)(0) \\
& +e^{-\left(\lambda_{0}+\delta_{0}\right) \tau} p_{2} v(t-\tau)-e^{-\lambda_{0} \tau} p_{2} \Phi_{1}\left(\lambda_{0} ; \phi\right)(-\tau)+\left(\delta_{0} p_{1}-3 \lambda_{0} \delta_{0}+q_{1}+2 p_{1} \lambda_{0}\right. \\
& \left.-3 \lambda_{0}^{2}-\delta_{0}^{2}\right) \int_{0}^{t} v(s) d s+e^{-\left(\lambda_{0}+\delta_{0}\right) \tau}\left(\delta_{0} p_{2}+q_{2}+2 p_{2} \lambda_{0}\right)\left\{\int_{-\tau}^{0} v(s) d s+\int_{0}^{t-\tau} v(s) d s\right\} \\
& -e^{-\lambda_{0} \tau}\left(p_{2} \lambda_{0}^{2}+q_{2} \lambda_{0}+v_{2}\right) \int_{0}^{\tau} e^{-\delta_{0} s}\left\{\int_{-s}^{0} v(u) d u+\int_{0}^{t-s} v(u) d u\right\} d s, \\
v^{\prime}(t)= & \left(p_{1}-3 \lambda_{0}-2 \delta_{0}\right) v(t)+e^{-\left(\lambda_{0}+\delta_{0}\right) \tau} p_{2} v(t-\tau) \\
& -\left\{e^{-\left(\lambda_{0}+\delta_{0}\right)}\left(p_{2} \delta_{0}+q_{2}+2 p_{2} \lambda_{0}\right)-\delta_{0}^{-1}\left(1-e^{-\delta_{0} \tau}\right)\left(p_{2} \lambda_{0}^{2}+q_{2} \lambda_{0}+v_{2}\right) e^{-\lambda_{0} \tau}\right\} \int_{0}^{t} v(s) d s \\
& +e^{-\left(\lambda_{0}+\delta_{0}\right) \tau}\left(\delta_{0} p_{2}+q_{2}+2 p_{2} \lambda_{0}\right) \int_{0}^{t-\tau} v(s) d s \\
& -e^{-\lambda_{0} \tau}\left(p_{2} \lambda_{0}^{2}+q_{2} \lambda_{0}+v_{2}\right) \int_{0}^{\tau} e^{-\delta_{0} s}\left\{\int_{0}^{t-s} v(u) d u\right\} d s+R\left(\lambda_{0}, \delta_{0} ; \phi\right), \\
& +e^{-\lambda_{0} \tau}\left(p_{2} \lambda_{0}^{2}+q_{2} \lambda_{0}+v_{2}\right) \int_{0}^{\tau} e^{-\delta_{0} s}\left\{\int_{t-s}^{t} v(u) d u\right\} d s+R\left(\lambda_{0}, \delta_{0} ; \phi\right), \\
v^{\prime}(t)= & \left(p_{1}-3 \lambda_{0}-2 \delta_{0}\right) v(t)+e^{-\left(\lambda_{0}+\delta_{0}\right) \tau} p_{2} v(t-\tau)-e^{-\left(\lambda_{0}+\delta_{0}\right) \tau}\left(p_{2} \delta_{0}+q_{2}+2 p_{2} \lambda_{0}\right) \int_{t-\tau}^{t} v(s) d s \\
& -e^{-\lambda_{0} \tau}\left(p_{2} \lambda_{0}^{2}+q_{2} \lambda_{0}+v_{2}\right) \int_{0}^{\tau} e^{-\delta_{0} s}\left\{\int_{0}^{t-s} v(u) d u\right\} d s+R\left(\lambda_{0}, \delta_{0} ; \phi\right), \\
v^{\prime}(t)= & \left(p_{1}-3 \lambda_{0}-2 \delta_{0}\right) v(t)+e^{-\left(\lambda_{0}+\delta_{0}\right) \tau} p_{2} v(t-\tau)-e^{-\left(\lambda_{0}+\delta_{0}\right) \tau}\left(p_{2} \delta_{0}+q_{2}+2 p_{2} \lambda_{0}\right) \int_{t-\tau}^{t} v(s) d s \\
& +e^{-\lambda_{0} \tau}\left(p_{2} \lambda_{0}^{2}+q_{2} \lambda_{0}+v_{2}\right) \int_{0}^{\tau} e^{-\delta_{0} s} d s \int_{0}^{t} v(s) d s \\
& \\
& \\
&
\end{aligned}
$$

where $R\left(\lambda_{0}, \delta_{0} ; \phi\right)$ was given in (20).

Next, we take into account the assumption $\eta_{\lambda_{0}, \delta_{0}} \neq 0$ and we define

$$
w(t)=v(t)-\frac{R\left(\lambda_{0}, \delta_{0} ; \phi\right)}{\eta_{\lambda_{0}, \delta_{0}}} \text { for } t \geq-\tau .
$$

Then, because of definition of $\eta_{\lambda_{0}, \delta_{0}}$ by (19), it is a matter of elementary calculations to show that $v$ satisfies (27) for $t \geq 0$ if and only if $w$ satisfies (17) for $t \geq 0$, i.e., if and only if $w$ is a solution of the delay differential equation (17). Moreover, we see that the initial condition $(26)$ is equivalently written as follows

$$
w(t)=e^{-\delta_{0} t} \Phi_{1}\left(\lambda_{0} ; \phi\right)(t)-\frac{R\left(\lambda_{0}, \delta_{0} ; \phi\right)}{\eta_{\lambda_{0}, \delta_{0}}} \quad \text { for } \quad-\tau \leq t \leq 0 .
$$

We have thus proved that $z$ is the solution of (4) and (10) if and only if $w$ is the solution of the delay differential equation (17) which satisfies the initial condition (29). By (24), we see that (28) coincides with (22). Also, by taking into account the definition of $\Phi_{2}\left(\lambda_{0}, \delta_{0} ; \phi\right)$ by (21), we observe that (29) coincides with the initial condition (23). The proof of the proposition 2 is completed.

Let $C([-\tau, 0], I R)$ be the Banach space of all continuous real-valued functions on the interval $[-\tau, 0]$, endowed with the usual sup-norm

$$
\|\Psi\|=\max _{-\tau \leq t \leq 0}|\Psi(t)| \quad \text { for } \quad \Psi \in C([-\tau, 0], I R) .
$$


Moreover, let $C^{2}([-\tau, 0], I R)$ be the set of all twice continuously differentiable real-valued functions on the interval $[-\tau, 0]$. This set is a Banach space with the norm

$$
\|\Psi\|_{C^{2}}=\max \left\{\|\Psi\|,\left\|\Psi^{\prime}\right\|,\left\|\Psi^{\prime \prime}\right\|\right\} \quad \text { for } \quad \Psi \in C^{2}([-\tau, 0], I R) .
$$

As it concerns the IVP (1) and (2) studied in this paper, the initial function $\phi$ belongs to $C^{2}([-\tau, 0], I R)$. So, the notation $\|\phi\|_{C^{2}}$ used in Section 3 is defined by

$$
\|\phi\|_{C^{2}}=\max \left\{\|\phi\|\left\|\phi^{\prime}\right\|\left\|\phi^{\prime \prime}\right\|\right\} \equiv \max \left\{\max _{-\tau \leq t \leq 0}|\phi(t)|, \max _{-\tau \leq t \leq 0}\left|\phi^{\prime}(t)\right|, \max _{-\tau \leq t \leq 0}\left|\phi^{\prime \prime}(t)\right|\right\} .
$$

Before closing this section, we will give three well-known definitions. The trivial solution of the delay differential equation (1) is said to be "stable" (at 0 ) if for every $\varepsilon>0$, there exists a $\ell=\ell(\varepsilon)>0$ such that, for any $\phi \in C^{2}([-\tau, 0], I R)$ with $\|\phi\|_{C^{2}}<\ell$, the solution $y$ of the IVP (1) and (2) satisfies

$$
|y(t)|<\varepsilon \text { for all } t \in[-\tau, \infty) .
$$

Otherwise, the trivial solution of (1) is said to be "unstable" (at 0). Moreover, the trivial solution of (1) is called "asymptotically stable" (at 0 ) if it is stable in the above sense and, in addition, there exists a $\ell_{0}>0$ such that, for any $\phi \in C^{2}([-\tau, 0], I R)$ with $\|\phi\|_{C^{2}}<\ell_{0}$, the solution $y$ of the IVP (1)-(2) satisfies

$$
\lim _{t \rightarrow \infty}|y(t)|=0 ; \quad \text { i.e., } \quad \lim _{t \rightarrow \infty} y(t)=0 .
$$

\section{An Asymptotic results}

Our purpose in this section is to establish the following theorem.

Theorem 1. Let $\lambda_{0}$ be real root of the characteristic equation (3), and let $\beta_{\lambda_{0}}$ and $L\left(\lambda_{0} ; \phi\right)$ be defined by (6) and (7), respectively. Furthermore, let $\delta_{0}$ be real root of the characteristic equation (5), and let $\eta_{\lambda_{0}, \delta_{0}}, R\left(\lambda_{0}, \delta_{0} ; \phi\right)$ and $\Phi_{2}\left(\lambda_{0}, \delta_{0} ; \phi\right)$ be defined by (19), (20) and (21), respectively. Moreover, let $\gamma_{0}$ be a real root of the characteristic equation (18). Suppose that $\beta_{\lambda_{0}} \neq 0$ and $\eta_{\lambda_{0}, \delta_{0}} \neq 0$. ( Note that, because of $\beta_{\lambda_{0}} \neq 0$, we always have $\delta_{0} \neq 0$ and $\gamma_{0} \neq-\delta_{0}$. Furthermore, because of $\eta_{\lambda_{0}, \delta_{0}} \neq 0$, we always have $\gamma_{0} \neq 0$.) Set

$$
\begin{aligned}
\xi_{\lambda_{0}, \delta_{0}, \gamma_{0}=} & e^{-\left(\lambda_{0}+\delta_{0}+\gamma_{0}\right) \tau} p_{2} \tau-e^{-\left(\lambda_{0}+\delta_{0}\right) \tau}\left(p_{2} \delta_{0}+q_{2}+2 p_{2} \lambda_{0}\right) \int_{0}^{\tau} e^{-\gamma_{0} s} s d s \\
& +e^{-\lambda_{0} \tau}\left(p_{2} \lambda_{0}^{2}+q_{2} \lambda_{0}+v_{2}\right) \int_{0}^{\tau} e^{-\delta_{0} s}\left\{\int_{0}^{s} e^{-\gamma_{0} u} u d u\right\} d s
\end{aligned}
$$

and, also, define

$$
\begin{aligned}
K\left(\lambda_{0}, \delta_{0}, \gamma_{0} ; \phi\right)= & \Phi_{2}\left(\lambda_{0}, \delta_{0} ; \phi\right)(0)+e^{-\left(\lambda_{0}+\delta_{0}+\gamma_{0}\right) \tau} p_{2} \int_{-\tau}^{0} e^{-\gamma_{0} s} \Phi_{2}\left(\lambda_{0}, \delta_{0} ; \phi\right)(s) d s \\
& -e^{-\left(\lambda_{0}+\delta_{0}\right) \tau}\left(p_{2} \delta_{0}+q_{2}+2 p_{2} \lambda_{0}\right) \int_{0}^{\tau} e^{-\gamma_{0} s}\left\{\int_{-s}^{0} e^{-\gamma_{0} u} \Phi_{2}\left(\lambda_{0}, \delta_{0} ; \phi\right)(u) d u\right\} d s \\
& +e^{-\lambda_{0} \tau}\left(p_{2} \lambda_{0}^{2}+q_{2} \lambda_{0}+v_{2}\right) \int_{0}^{\tau} e^{-\delta_{0} s}\left\{\int_{0}^{s} e^{-\gamma_{0} u}\left\{\int_{-u}^{0} e^{-\gamma_{0} \omega} \Phi_{2}\left(\lambda_{0}, \delta_{0} ; \phi\right)(\omega) d \omega\right\} d u\right\} d s .
\end{aligned}
$$

Assume that

$$
\begin{aligned}
\mu_{\lambda_{0}, \delta_{0}, \gamma_{0}}= & e^{-\left(\lambda_{0}+\delta_{0}+\gamma_{0}\right) \tau}\left|p_{2}\right| \tau+e^{-\left(\lambda_{0}+\delta_{0}\right) \tau}\left|p_{2} \delta_{0}+q_{2}+2 p_{2} \lambda_{0}\right| \int_{0}^{\tau} e^{-\gamma_{0} s} s d s \\
& +e^{-\lambda_{0} \tau}\left|p_{2} \lambda_{0}^{2}+q_{2} \lambda_{0}+v_{2}\right| \int_{0}^{\tau} e^{-\delta_{0} s}\left\{\int_{0}^{s} e^{-\gamma_{0} u} u d u\right\} d s<1
\end{aligned}
$$


( This assumption guarantees that $1+\xi_{\lambda_{0}, \delta_{0}, \gamma_{0}}>0$.) Then the solution $y$ of the IVP (1) and (2) satisfies

$$
\lim _{t \rightarrow \infty}\left\{e^{-\gamma_{0} t}\left[e^{-\left(\lambda_{0}+\delta_{0}\right) t} y(t)-\frac{L\left(\lambda_{0} ; \phi\right)}{\beta_{\lambda_{0}}} e^{-\delta_{0} t}-\frac{R\left(\lambda_{0}, \delta_{0} ; \phi\right)}{\eta_{\lambda_{0}, \delta_{0}}}\right]\right\}=\frac{K\left(\lambda_{0}, \delta_{0}, \gamma_{0} ; \phi\right)}{1+\xi_{\lambda_{0}, \delta_{0}, \gamma_{0}}}
$$

Before we prove the above theorem, we will present some observations, which are concerned with a real root $\lambda_{0}$ of the characteristic equation (3), a real root $\delta_{0}$ of the characteristic equation (5) and a real root $\gamma_{0}$ of the characteristic equation (18).

Let $F(\delta)$ denote the characteristic function of (5), i.e.,

$$
\begin{aligned}
F(\delta)= & \delta^{2}-\left(p_{1}-3 \lambda_{0}\right) \delta-p_{2} \delta e^{-\left(\lambda_{0}+\delta\right) \tau}-q_{1}-2 p_{1} \lambda_{0}+3 \lambda_{0}^{2} \\
& -\left(q_{2}+2 p_{2} \lambda_{0}\right) e^{-\left(\lambda_{0}+\delta\right) \tau}+e^{-\lambda_{0} \tau}\left(p_{2} \lambda_{0}^{2}+q_{2} \lambda_{0}+v_{2}\right) \delta^{-1}\left(1-e^{-\delta \tau}\right) .
\end{aligned}
$$

Since $\delta=0$ is a removable singularity of $F(\delta)$, we can regard $F(\delta)$ as a entire function with

$$
F(0)=e^{-\lambda_{0} \tau}\left(\left(p_{2} \lambda_{0}^{2}+q_{2} \lambda_{0}+v_{2}\right) \tau-q_{2}-2 p_{2} \lambda_{0}\right)-q_{1}-2 p_{1} \lambda_{0}+3 \lambda_{0}^{2} \equiv \beta_{\lambda_{0}}
$$

Hence, if we assume that $\beta_{\lambda_{0}} \neq 0$, then we always have $\delta_{0} \neq 0$. Let $G(\gamma)$ denote the characteristic function of (18), i.e.,

$$
\begin{aligned}
G(\gamma)= & -\gamma+p_{1}-3 \lambda_{0}-2 \delta_{0}+p_{2} e^{-\left(\lambda_{0}+\delta_{0}+\gamma\right) \tau}-e^{-\left(\lambda_{0}+\delta_{0}\right) \tau}\left(p_{2} \delta_{0}+q_{2}+2 p_{2} \lambda_{0}\right) \gamma^{-1}\left(1-e^{-\gamma \tau}\right) \\
& +e^{-\lambda_{0} \tau}\left(p_{2} \lambda_{0}^{2}+q_{2} \lambda_{0}+v_{2}\right) \gamma^{-1}\left\{\delta_{0}^{-1}\left(1-e^{-\delta_{0} \tau}\right)-\left(\delta_{0}+\gamma\right)^{-1}\left(1-e^{-\left(\delta_{0}+\gamma\right) \tau}\right)\right\} .
\end{aligned}
$$

Since $\gamma=0$ is a removable singularity of $G(\gamma)$, we can regard $G(\gamma)$ as a entire function with

$$
\begin{aligned}
G(0)= & p_{1}-3 \lambda_{0}-2 \delta_{0}+p_{2} e^{-\left(\lambda_{0}+\delta_{0}\right) \tau}-e^{-\left(\lambda_{0}+\delta_{0}\right) \tau}\left(p_{2} \delta_{0}+q_{2}+2 p_{2} \lambda_{0}\right) \tau \\
& +e^{-\lambda_{0} \tau}\left(p_{2} \lambda_{0}^{2}+q_{2} \lambda_{0}+v_{2}\right) \delta_{0}^{-2}\left(1-e^{-\delta_{0} \tau}-\delta_{0} \tau e^{-\delta_{0} \tau}\right) \equiv \eta_{\lambda_{0}, \delta_{0}}
\end{aligned}
$$

Hence, if we assume that $\eta_{\lambda_{0}, \delta_{0}} \neq 0$, then we always have $\gamma_{0} \neq 0$. Furthermore, since $\gamma=-\delta_{0}\left(\delta_{0} \neq 0\right)$ is a removable singularity of $G(\gamma)$, we can regard $G(\gamma)$ as a entire function with

$$
\begin{aligned}
G\left(-\delta_{0}\right)= & \delta_{0}+p_{1}-3 \lambda_{0}-2 \delta_{0}+p_{2} e^{-\lambda_{0} \tau}+e^{-\left(\lambda_{0}+\delta_{0}\right) \tau}\left(p_{2} \delta_{0}+q_{2}+2 p_{2} \lambda_{0}\right) \delta_{0}^{-1}\left(1-e^{\delta_{0} \tau}\right) \\
& -e^{-\lambda_{0} \tau}\left(p_{2} \lambda_{0}^{2}+q_{2} \lambda_{0}+v_{2}\right) \delta_{0}^{-1}\left(\delta_{0}^{-1}\left(1-e^{-\delta_{0} \tau}\right)-\tau\right)
\end{aligned}
$$

or

$$
\begin{aligned}
\delta_{0} G\left(-\delta_{0}\right)= & -\delta_{0}^{2}+\delta_{0}\left(p_{1}-3 \lambda_{0}\right)+\delta_{0} p_{2} e^{-\lambda_{0} \tau}+e^{-\left(\lambda_{0}+\delta_{0}\right) \tau}\left(p_{2} \delta_{0}+q_{2}+2 p_{2} \lambda_{0}\right) \\
& -e^{-\lambda_{0} \tau}\left(p_{2} \delta_{0}+q_{2}+2 p_{2} \lambda_{0}\right)-e^{-\lambda_{0} \tau}\left(p_{2} \lambda_{0}^{2}+q_{2} \lambda_{0}+v_{2}\right) \delta_{0}^{-1}\left(1-e^{-\delta_{0} \tau}\right)+e^{-\lambda_{0} \tau}\left(p_{2} \lambda_{0}^{2}+q_{2} \lambda_{0}+v_{2}\right) \tau
\end{aligned}
$$

By using (5), we derive

$$
\delta_{0} G\left(-\delta_{0}\right)=-\left(q_{1}+2 p_{1} \lambda_{0}-3 \lambda_{0}^{2}\right)-e^{-\lambda_{0} \tau}\left(q_{2}+2 p_{2} \lambda_{0}\right)+e^{-\lambda_{0} \tau}\left(p_{2} \lambda_{0}^{2}+q_{2} \lambda_{0}+v_{2}\right) \tau \equiv \beta_{\lambda_{0}} .
$$

But, by the definition of $\beta_{\lambda_{0}} \neq 0$, a root of the characteristic equation (18) must become $\gamma_{0} \neq-\delta_{0}$. Consequently, it must be $\delta_{0} \neq 0$ real root of the characteristic equation (5) and $\gamma_{0} \neq 0, \gamma_{0} \neq-\delta_{0}$ real root of the characteristic equation (18).

Define $\mu_{\lambda_{0}, \delta_{0}, \gamma_{0}}$ by (32). It is clear $\mu_{\lambda_{0}, \delta_{0}, \gamma_{0}}$ is positive. So, (32) can equivalently be written as follows

$$
0<\mu_{\lambda_{0}, \delta_{0}, \gamma_{0}}<1
$$


Furthermore, for the real constant $\xi_{\lambda_{0}, \delta_{0}, \gamma_{0}}$ defined by (30), we have

$$
\begin{aligned}
\left|\xi_{\lambda_{0}, \delta_{0}, \gamma_{0}}\right| \leq & \left|e^{-\left(\lambda_{0}+\delta_{0}+\gamma_{0}\right) \tau} p_{2} \tau\right|+\left|e^{-\left(\lambda_{0}+\delta_{0}\right) \tau}\left(p_{2} \delta_{0}+q_{2}+2 p_{2} \lambda_{0}\right) \int_{0}^{\tau} e^{-\gamma_{0} s} s d s\right| \\
& +\left|e^{-\lambda_{0} \tau}\left(p_{2} \lambda_{0}^{2}+q_{2} \lambda_{0}+v_{2}\right) \int_{0}^{\tau} e^{-\delta_{0} s}\left\{\int_{0}^{s} e^{-\gamma_{0} u} u d u\right\} d s\right| \\
& \leq e^{-\left(\lambda_{0}+\delta_{0}+\gamma_{0}\right) \tau}\left|p_{2}\right| \tau+e^{-\left(\lambda_{0}+\delta_{0}\right) \tau}\left|p_{2} \delta_{0}+q_{2}+2 p_{2} \lambda_{0}\right| \int_{0}^{\tau} e^{-\gamma_{0} s} s d s \\
& +e^{-\lambda_{0} \tau}\left|p_{2} \lambda_{0}^{2}+q_{2} \lambda_{0}+v_{2}\right| \int_{0}^{\tau} e^{-\delta_{0} s}\left\{\int_{0}^{s} e^{-\gamma_{0} u} u d u\right\} d s \equiv \mu_{\lambda_{0}, \delta_{0}, \gamma_{0}}
\end{aligned}
$$

That is,

$$
\left|\xi_{\lambda_{0}, \delta_{0}, \gamma_{0}}\right| \leq \mu_{\lambda_{0}, \delta_{0}, \gamma_{0}}
$$

Thus, if we assume that (32) is satisfied, i.e., that (34) holds, then (35) gives $\left|\xi_{\lambda_{0}, \delta_{0}, \gamma_{0}}\right|<1$. This guarantees, in particular, that

$$
1+\xi_{\lambda_{0}, \delta_{0}, \gamma_{0}}>0
$$

Proof. Let $y$ be the solution of the IVP (1) and (2). Define the function $z$ by (9). By Proposition 1.1, the fact that $y$ is the solution of the IVP (1) and (2) is equivalent to the fact that $z$ is the solution of the delay differential equation (4) which satisfies the initial condition (10). Furthermore, define the function $w$ by (22). By Proposition 2 , the fact that $z$ is the solution of the delay differential equation (4) and (10) is equivalent to the fact that $w$ is the solution of the delay differential equation (17) which satisfies the initial condition (23). Set

$$
\Omega(t)=e^{-\gamma_{0} t} w(t) \text { for all } t \in[-\tau, \infty)
$$

Then, using the fact that $\gamma_{0}$ is a real root of the characteristic equation (18), from (17) we obtain, for every $t \geq 0$,

$$
\begin{aligned}
\Omega^{\prime}(t)= & \left(p_{1}-3 \lambda_{0}-2 \delta_{0}-\gamma_{0}\right) \Omega(t)+e^{-\left(\lambda_{0}+\delta_{0}+\gamma_{0}\right) \tau} p_{2} \Omega(t-\tau) \\
& \left.-e^{-\left(\lambda_{0}+\delta_{0}\right) \tau}\left(p_{2} \delta_{0}+q_{2}+2 p_{2} \lambda_{0}\right)\right) \int_{0}^{\tau} e^{-\gamma_{0} s} \Omega(t-s) d s \\
& +e^{-\lambda_{0} \tau}\left(p_{2} \lambda_{0}^{2}+q_{2} \lambda_{0}+v_{2}\right) \int_{0}^{\tau} e^{-\delta_{0} s}\left\{\int_{0}^{s} e^{-\gamma_{0} u} \Omega(t-u) d u\right\} d s .
\end{aligned}
$$

Moreover, the initial condition (29) can be equivalently written

$$
\Omega(t)=e^{-\gamma_{0} t} \Phi_{2}\left(\lambda_{0}, \delta_{0} ; \phi\right)(t) \text { for } t \in[-\tau, 0]
$$

where $\Phi_{2}\left(\lambda_{0}, \delta_{0} ; \phi\right)$ is defined by (21). Furthermore, using the fact that $\gamma_{0}$ is a real root of (18) and taking into account (38), we can verify that (37) is equivalent to

$$
\begin{aligned}
\Omega(t)= & \left(p_{1}-3 \lambda_{0}-2 \delta_{0}-\gamma_{0}\right) \int_{0}^{t} \Omega(s) d s+e^{-\left(\lambda_{0}+\delta_{0}+\gamma_{0}\right) \tau} p_{2} \int_{0}^{t} \Omega(s-\tau) d s \\
& \left.-e^{-\left(\lambda_{0}+\delta_{0}\right) \tau}\left(p_{2} \delta_{0}+q_{2}+2 p_{2} \lambda_{0}\right)\right) \int_{0}^{\tau} e^{-\gamma_{0} s}\left\{\int_{0}^{t} \Omega(u-s) d u\right\} d s \\
& +e^{-\lambda_{0} \tau}\left(p_{2} \lambda_{0}^{2}+q_{2} \lambda_{0}+v_{2}\right) \int_{0}^{\tau} e^{-\delta_{0} s}\left\{\int_{0}^{s} e^{-\gamma_{0} u}\left\{\int_{0}^{t} \Omega(\omega-u) d \omega\right\} d u\right\} d s+\Omega(0),
\end{aligned}
$$




$$
\begin{gathered}
\Omega(t)=\left(p_{1}-3 \lambda_{0}-2 \delta_{0}-\gamma_{0}\right) \int_{0}^{t} \Omega(s) d s+e^{-\left(\lambda_{0}+\delta_{0}+\gamma_{0}\right) \tau} p_{2} \int_{-\tau}^{t-\tau} \Omega(s) d s \\
\left.\quad-e^{-\left(\lambda_{0}+\delta_{0}\right) \tau}\left(p_{2} \delta_{0}+q_{2}+2 p_{2} \lambda_{0}\right)\right) \int_{0}^{\tau} e^{-\gamma_{0} s}\left\{\int_{-s}^{t-s} \Omega(u) d u\right\} d s \\
+e^{-\lambda_{0} \tau}\left(p_{2} \lambda_{0}^{2}+q_{2} \lambda_{0}+v_{2}\right) \int_{0}^{\tau} e^{-\delta_{0} s}\left\{\int_{0}^{s} e^{-\gamma_{0} u}\left\{\int_{-u}^{t-u} \Omega(\omega) d \omega\right\} d u\right\} d s+\Omega(0), \\
\Omega(t)=-e^{-\left(\lambda_{0}+\delta_{0}+\gamma_{0}\right) \tau} p_{2} \int_{t-\tau}^{t} \Omega(s) d s+e^{-\left(\lambda_{0}+\delta_{0}\right) \tau}\left(p_{2} \delta_{0}+q_{2}+2 p_{2} \lambda_{0}\right) \int_{0}^{\tau} e^{-\gamma_{0} s}\left\{\int_{t-s}^{t} \Omega(u) d u\right\} d s \\
-e^{-\lambda_{0} \tau}\left(p_{2} \lambda_{0}^{2}+q_{2} \lambda_{0}+v_{2}\right) \int_{0}^{\tau} e^{-\delta_{0} s}\left\{\int_{0}^{s} e^{-\gamma_{0} u}\left\{\int_{t-u}^{t} \Omega(\omega) d \omega\right\} d u\right\} d s+K\left(\lambda_{0}, \delta_{0}, \gamma_{0} ; \phi\right),
\end{gathered}
$$

where $K\left(\lambda_{0}, \delta_{0}, \gamma_{0} ; \phi\right)$ is defined by (31). Next, we define

$$
\Theta(t)=\Omega(t)-\frac{K\left(\lambda_{0}, \delta_{0}, \gamma_{0} ; \phi\right)}{1+\xi_{\lambda_{0}, \delta_{0}, \gamma_{0}}}, \quad \text { for } \quad t \geq-\tau
$$

where $\xi_{\lambda_{0}, \delta_{0}, \gamma_{0}}$ is defined by (30). Then we can see that (39) reduces to the following equivalent equation

$$
\begin{aligned}
\Theta(t)= & -e^{-\left(\lambda_{0}+\delta_{0}+\gamma_{0}\right) \tau} p_{2} \int_{t-\tau}^{t} \Theta(s) d s+e^{-\left(\lambda_{0}+\delta_{0}\right) \tau}\left(p_{2} \delta_{0}+q_{2}+2 p_{2} \lambda_{0}\right) \int_{0}^{\tau} e^{-\gamma_{0} s}\left\{\int_{t-s}^{t} \Theta(u) d u\right\} d s \\
& -e^{-\lambda_{0} \tau}\left(p_{2} \lambda_{0}^{2}+q_{2} \lambda_{0}+v_{2}\right) \int_{0}^{\tau} e^{-\delta_{0} s}\left\{\int_{0}^{s} e^{-\gamma_{0} u}\left\{\int_{t-u}^{t} \Theta(\omega) d \omega\right\} d u\right\} d s
\end{aligned}
$$

for all $t \geq 0$. On the other hand, the initial condition (38) can be equivalently written

$$
\Theta(t)=e^{-\gamma_{0} t} \Phi_{2}\left(\lambda_{0}, \delta_{0} ; \phi\right)(t)-\frac{K\left(\lambda_{0}, \delta_{0}, \gamma_{0} ; \phi\right)}{1+\xi_{\lambda_{0}, \delta_{0}, \gamma_{0}}}, \quad \text { for } \quad t \in[-\tau, 0]
$$

Now, we shall prove that

$$
\lim _{t \rightarrow \infty} \Theta(t)=0
$$

Define

$$
M\left(\lambda_{0}, \delta_{0}, \gamma_{0} ; \phi\right)=\max _{-\tau \leq t \leq 0}\left|e^{-\gamma_{0} t} \Phi_{2}\left(\lambda_{0}, \delta_{0} ; \phi\right)(t)-\frac{K\left(\lambda_{0}, \delta_{0}, \gamma_{0} ; \phi\right)}{1+\xi_{\lambda_{0}, \delta_{0}, \gamma_{0}}}\right|
$$

It follows from (42) and (44) that

$$
|\Theta(t)| \leq M\left(\lambda_{0}, \delta_{0}, \gamma_{0} ; \phi\right) \text { for } \quad-\tau \leq t \leq 0
$$

We will show that $M\left(\lambda_{0}, \delta_{0}, \gamma_{0} ; \phi\right)$ is a bound of the function $\Theta$ on the whole interval $[-\tau, \infty)$, i.e., that

$$
|\Theta(t)| \leq M\left(\lambda_{0}, \delta_{0}, \gamma_{0} ; \phi\right) \text { for all } t \geq-\tau
$$

For this purpose, we consider an arbitrary positive real number $\varepsilon$. We claim that 


$$
|\Theta(t)|<M\left(\lambda_{0}, \delta_{0}, \gamma_{0} ; \phi\right)+\varepsilon \text { for every } t \geq-\tau
$$

Otherwise, since (45) implies that $|\Theta(t)|<M\left(\lambda_{0}, \delta_{0}, \gamma_{0} ; \phi\right)+\varepsilon$ for $\quad-\tau \leq t \leq 0$, there exists a point $t^{*}>0$ such that

$$
|\Theta(t)|<M\left(\lambda_{0}, \delta_{0}, \gamma_{0} ; \phi\right)+\varepsilon, \quad \text { for } \quad-\tau \leq t<t^{*} \quad \text { and } \quad\left|\Theta\left(t^{*}\right)\right|=M\left(\lambda_{0}, \delta_{0}, \gamma_{0} ; \phi\right)+\varepsilon .
$$

Then, by taking into account the definition of $\mu_{\lambda_{0}, \delta_{0}, \gamma_{0}}$ by (32) and using (34), from (41) we obtain

$$
\begin{aligned}
M\left(\lambda_{0}, \delta_{0}, \gamma_{0} ; \phi\right)+\varepsilon= & \left|\Theta\left(t^{*}\right)\right| \\
\leq & e^{-\left(\lambda_{0}+\delta_{0}+\gamma_{0}\right) \tau}\left|p_{2}\right| \int_{t^{*}-\tau}^{t^{*}}|\Theta(s)| d s+e^{-\left(\lambda_{0}+\delta_{0}\right) \tau}\left|p_{2} \delta_{0}+q_{2}+2 p_{2} \lambda_{0}\right| \int_{0}^{\tau} e^{-\gamma_{0} s}\left\{\int_{t^{*}-s}^{t^{*}}|\Theta(u)| d u\right\} d s \\
& +e^{-\lambda_{0} \tau}\left|p_{2} \lambda_{0}^{2}+q_{2} \lambda_{0}+v_{2}\right| \int_{0}^{\tau} e^{-\delta_{0} s}\left\{\int_{0}^{s} e^{-\gamma_{0} u}\left\{\int_{t^{*}-u}^{t^{*}}|\Theta(\omega)| d \omega\right\} d u\right\} d s \\
\leq & \left\{e^{-\left(\lambda_{0}+\delta_{0}+\gamma_{0}\right) \tau}\left|p_{2}\right| \tau+e^{-\left(\lambda_{0}+\delta_{0}\right) \tau}\left|p_{2} \delta_{0}+q_{2}+2 p_{2} \lambda_{0}\right| \int_{0}^{\tau} e^{-\gamma_{0} s} s d s\right. \\
& \left.+e^{-\lambda_{0} \tau}\left|p_{2} \lambda_{0}^{2}+q_{2} \lambda_{0}+v_{2}\right| \int_{0}^{\tau} e^{-\delta_{0} s}\left\{\int_{0}^{s} e^{-\gamma_{0} u} u d u\right\} d s\right\}\left(M\left(\lambda_{0}, \delta_{0}, \gamma_{0} ; \phi\right)+\varepsilon\right) \\
= & \mu_{\lambda_{0}, \delta_{0}, \gamma_{0}} M\left(\lambda_{0}, \delta_{0}, \gamma_{0} ; \phi\right) \\
< & M\left(\lambda_{0}, \delta_{0}, \gamma_{0} ; \phi\right)+\varepsilon .
\end{aligned}
$$

We have thus arrived at a contradiction, which establishes our claim, i.e., that (47) holds true. As (47) is satisfied for all real numbers $\varepsilon>0$, it follows that (46) is always fulfilled. Furthermore, by using (46), from (41) we get, for every $t \geq 0$,

$$
\begin{aligned}
|\Theta(t)| \leq & e^{-\left(\lambda_{0}+\delta_{0}+\gamma_{0}\right) \tau} p_{2} \int_{t-\tau}^{t}|\Theta(s)| d s+e^{-\left(\lambda_{0}+\delta_{0}\right) \tau}\left|p_{2} \delta_{0}+q_{2}+2 p_{2} \lambda_{0}\right| \int_{0}^{\tau} e^{-\gamma_{0} s}\left\{\int_{t-s}^{t}|\Theta(u)| d u\right\} d s \\
& +e^{-\lambda_{0} \tau}\left|p_{2} \lambda_{0}^{2}+q_{2} \lambda_{0}+v_{2}\right| \int_{0}^{\tau} e^{-\delta_{0} s}\left\{\int_{0}^{s} e^{-\gamma_{0} u}\left\{\int_{t-u}^{t}|\Theta(\omega)| d \omega\right\} d u\right\} d s \\
\leq & \left\{e^{-\left(\lambda_{0}+\delta_{0}+\gamma_{0}\right) \tau}\left|p_{2}\right| \tau+e^{-\left(\lambda_{0}+\delta_{0}\right) \tau}\left|p_{2} \delta_{0}+q_{2}+2 p_{2} \lambda_{0}\right| \int_{0}^{\tau} e^{-\gamma_{0} s} s d s\right. \\
& \left.+e^{-\lambda_{0} \tau}\left|p_{2} \lambda_{0}^{2}+q_{2} \lambda_{0}+v_{2}\right| \int_{0}^{\tau} e^{-\delta_{0} s}\left\{\int_{0}^{s} e^{-\gamma_{0} u} u d u\right\} d s\right\} M\left(\lambda_{0}, \delta_{0}, \gamma_{0} ; \phi\right) .
\end{aligned}
$$

Thus, by taking into account the definition of $\mu_{\lambda_{0}, \delta_{0}, \gamma_{0}}$, by (32), we have

$$
|\Theta(t)| \leq \mu_{\lambda_{0}, \delta_{0}, \gamma_{0}} M\left(\lambda_{0}, \delta_{0}, \gamma_{0} ; \phi\right) \text { for every } t \geq 0
$$

By using (41) and taking into account the definition of $\mu_{\lambda_{0}, \delta_{0}, \gamma_{0}}$ by (32) as well as taking into account (46) and (48), one can prove, by an easy induction, that the function $\Theta$ satisfies

$$
|\Theta(t)| \leq\left(\mu_{\lambda_{0}, \delta_{0}, \gamma_{0}}\right){ }^{n} M\left(\lambda_{0}, \delta_{0}, \gamma_{0} ; \phi\right), \quad \text { for all } \quad t \geq n \tau-\tau,(n=0,1, \ldots)
$$

Because of (34), we have

$$
\lim _{n \rightarrow \infty}\left[\mu_{\lambda_{0}, \delta_{0}, \gamma_{0}}\right]^{n}=0
$$


In view of (50), it follows from (49) that $\lim _{t \rightarrow \infty} \Theta(t)=0$, i.e., (43) holds true.

Finally, by (22), (36) and (40), we have

$$
\Theta(t)=e^{-\gamma_{0} t}\left[e^{-\left(\lambda_{0}+\delta_{0}\right) t} y(t)-\frac{L\left(\lambda_{0} ; \phi\right)}{\beta_{\lambda_{0}}} e^{-\delta_{0} t}-\frac{R\left(\lambda_{0}, \delta_{0} ; \phi\right)}{\eta_{\lambda_{0}, \delta_{0}}}\right]-\frac{K\left(\lambda_{0}, \delta_{0}, \gamma_{0} ; \phi\right)}{1+\xi_{\lambda_{0}, \delta_{0}, \gamma_{0}}}
$$

for $t \geq-\tau$. In view of this equality, (33) coincides with (43). So, the solution $y$ of the IVP (1) and (2) satisfies (33). The proof of the theorem is complete.

\section{An estimate of the solutions and a stability criterion}

Our results in this section are Theorem 3.1 below and its corollary.

Theorem 2. Let $\lambda_{0}$ be a real root of the characteristic equation (3), and suppose that $\beta_{\lambda_{0}} \neq 0$, where $\beta_{\lambda_{0}}$ is defined by (6). Let $\delta_{0}$ be a real root of the characteristic equation (5), suppose that $\eta_{\lambda_{0}, \delta_{0}} \neq 0$, where $\eta_{\lambda_{0}, \delta_{0}}$ is defined by (19). Furthermore, let $\gamma_{0}$ be a real root of the characteristic equation (18), and let $\xi_{\lambda_{0}, \delta_{0}, \gamma_{0}}$ be defined by (30). ( Note that, because of $\beta_{\lambda_{0}} \neq 0$, we always have $\delta_{0} \neq 0$ and $\gamma_{0} \neq-\delta_{0}$. Furthermore, because of $\eta_{\lambda_{0}, \delta_{0}} \neq 0$, we always have $\gamma_{0} \neq 0$. ) Set

$$
m\left(\lambda_{0}, \delta_{0}, \gamma_{0}\right)=\max \left\{1, e^{\lambda_{0} \tau}, e^{\delta_{0} \tau}, e^{\gamma_{0} \tau}, e^{\left(\lambda_{0}+\delta_{0}\right) \tau}\right\}
$$

Assume that $\mu_{\lambda_{0}, \delta_{0}, \gamma_{0}}<1$ holds, where $\mu_{\lambda_{0}, \delta_{0}, \gamma_{0}}$ is defined by (32). ( This assumption guarantees that $1+\xi_{\lambda_{0}, \delta_{0}, \gamma_{0}}>0$. ) Then the solution $y$ of the IVP (1) and (2) satisfies

$$
|y(t)| \leq\left\{\frac{k_{\lambda_{0}}}{\left|\beta_{\lambda_{0}}\right|} e^{\lambda_{0} t}+\frac{m\left(\lambda_{0}, \delta_{0}, \gamma_{0}\right) h_{\lambda_{0}, \delta_{0}}}{\left|\eta_{\lambda_{0}, \delta_{0}}\right|} e^{\left(\lambda_{0}+\delta_{0}\right) t}+m\left(\lambda_{0}, \delta_{0}, \gamma_{0}\right) g\left(\lambda_{0}, \delta_{0}, \gamma_{0}\right) d_{\lambda_{0}, \delta_{0}} e^{\left(\lambda_{0}+\delta_{0}+\gamma_{0}\right) t}\right\}\|\phi\|_{C^{2}}
$$

for all $t \geq 0$, where

$$
\begin{gathered}
k_{\lambda_{0}}=1+\left|\lambda_{0}-p_{1}\right|+\left|p_{2}\right|+\left|\lambda_{0}^{2}-p_{1} \lambda_{0}-q_{1}\right|+\left|p_{2} \lambda_{0}+q_{2}\right|+e^{-\lambda_{0} \tau}\left|p_{2} \lambda_{0}^{2}+q_{2} \lambda_{0}+v_{2}\right| \int_{-\tau}^{0} e^{-\lambda_{0} s} d s, \\
h_{\lambda_{0}, \delta_{0}}=\left(1+\frac{k_{\lambda_{0}}}{\left|\beta_{\lambda_{0}}\right|}\right)\left\{\left(1+\left|\lambda_{0}\right|+\left|\delta_{0}\right|\right)+\left|p_{1}-3 \lambda_{0}-2 \delta_{0}\right|+e^{-\lambda_{0} \tau}\left|p_{2}\right|\right. \\
\left.+e^{-\left(\lambda_{0}+\delta_{0}\right) \tau}\left|p_{2} \delta_{0}+q_{2}+2 p_{2} \lambda_{0}\right| \int_{-\tau}^{0} e^{-\delta_{0} s} d s\right\} \\
d_{\lambda_{0}, \delta_{0}}=1+\frac{k_{\lambda_{0}}}{\left|\beta_{\lambda_{0}}\right|}+\frac{h_{\lambda_{0}, \delta_{0}}}{\left|\eta_{\lambda_{0}, \delta_{0}}\right|} \\
g\left(\lambda_{0}, \delta_{\left.0, \gamma_{0}\right)=} \mu_{\lambda_{0}, \delta_{0}, \gamma_{0}} m\left(\lambda_{0}, \delta_{0}, \gamma_{0}\right)+\frac{1+\mu_{\lambda_{0}, \delta_{0}, \gamma_{0}}}{1+\xi_{\lambda_{0}, \delta_{0}, \gamma_{0}}} \ell_{\lambda_{0}, \delta_{0}, \gamma_{0}},\right.
\end{gathered}
$$


and

$$
\begin{aligned}
\ell_{\lambda_{0}, \delta_{0}, \gamma_{0}}= & \left\{1+e^{-\left(\lambda_{0}+\delta_{0}+\gamma_{0}\right) \tau}\left|p_{2}\right| \int_{-\tau}^{0} e^{-\gamma_{0} s} d s+e^{-\left(\lambda_{0}+\delta_{0}\right) \tau}\left|p_{2} \delta_{0}+q_{2}+2 p_{2} \lambda_{0}\right| \int_{0}^{\tau} e^{-\gamma_{0} s}\left\{\int_{-s}^{0} e^{-\gamma_{0} u} d u\right\} d s\right. \\
& \left.+e^{-\lambda_{0} \tau}\left|p_{2} \lambda_{0}^{2}+q_{2} \lambda_{0}+v_{2}\right| \int_{0}^{\tau} e^{-\delta_{0} s}\left\{\int_{0}^{s} e^{-\gamma_{0} u}\left\{\int_{-u}^{0} e^{-\gamma_{0} \omega} d \omega\right\} d u\right\} d s\right\} .
\end{aligned}
$$

The constant $g\left(\lambda_{0}, \delta_{0}, \gamma_{0}\right)$ is greater than 1 .

Corollary 1. Let $\lambda_{0}, \delta_{0}$ and $\gamma_{0}$ be real roots of the characteristic equations (3), (5) and (18), respectively. Suppose that $\beta_{\lambda_{0}} \neq 0$ and $\eta_{\lambda_{0}, \delta_{0}} \neq 0$, where $\beta_{\lambda_{0}}$ and $\eta_{\lambda_{0}, \delta_{0}}$ are defined by (6) and (19), respectively. (Note that, because of $\beta_{\lambda_{0}} \neq 0$, we always have $\delta_{0} \neq 0$ and $\gamma_{0} \neq-\delta_{0}$. Furthermore, because of $\eta_{\lambda_{0}, \delta_{0}} \neq 0$, we always have $\left.\gamma_{0} \neq 0\right)$.

Assume that $\mu_{\lambda_{0}, \delta_{0}, \gamma_{0}}<1$ holds, where $\mu_{\lambda_{0}, \delta_{0}, \gamma_{0}}$ is defined by (32). Then the trivial solution of the delay differential equation (1) is stable if $\lambda_{0} \leq 0, \lambda_{0}+\delta_{0} \leq 0, \lambda_{0}+\delta_{0}+\gamma_{0} \leq 0$ it is asymptotically stable if $\lambda_{0}<0, \lambda_{0}+\delta_{0}<0$, $\lambda_{0}+\delta_{0}+\gamma_{0}<0$.

Proof. (Proof of Theorem 2.) First of all, we observe that, for any real number $a$, it holds $\max _{-\tau \leq t \leq 0} e^{-a t}=\max \left\{1, e^{a \tau}\right\}$. So, using (52), we immediately see that for $-\tau \leq t \leq 0$

$$
\begin{aligned}
e^{-\lambda_{0} t} & \leq m\left(\lambda_{0}, \delta_{0}, \gamma_{0}\right), \\
e^{-\delta_{0} t} & \leq m\left(\lambda_{0}, \delta_{0}, \gamma_{0}\right), \\
e^{-\gamma_{0} t} & \leq m\left(\lambda_{0}, \delta_{0}, \gamma_{0}\right), \\
e^{-\left(\lambda_{0}+\delta_{0}\right) t} & \leq m\left(\lambda_{0}, \delta_{0}, \gamma_{0}\right) .
\end{aligned}
$$

These inequalities will be frequently used later.

Define $L\left(\lambda_{0} ; \phi\right)$ by (7). Then

$$
\begin{aligned}
\left|L\left(\lambda_{0} ; \phi\right)\right| \leq & \left|\phi^{\prime \prime}(0)\right|+\left|\lambda_{0}-p_{1}\right|\left|\phi^{\prime}(0)\right|+\left|p_{2}\right|\left|\phi^{\prime}(-\tau)\right|+\left|\lambda_{0}^{2}-p_{1} \lambda_{0}-q_{1}\right||\phi(0)| \\
& +\left|p_{2} \lambda_{0}+q\right||\phi(-\tau)|+e^{-\lambda_{0} \tau}\left|p_{2} \lambda_{0}^{2}+q_{2} \lambda_{0}+v_{2}\right| \int_{-\tau}^{0} e^{-\lambda_{0} s}|\phi(s)| d s \\
\leq & \left\{\left\|\phi^{\prime \prime}\right\|+\left(\left|\lambda_{0}-p_{1}\right|+\left|p_{2}\right|\right)\left\|\phi^{\prime}\right\|+\left(\left|\lambda_{0}^{2}-p_{1} \lambda_{0}-q_{1}\right|+\left|p_{2} \lambda_{0}+q_{2}\right|\right.\right. \\
& \left.+e^{-\lambda_{0} \tau}\left|p_{2} \lambda_{0}^{2}+q_{2} \lambda_{0}+v_{2}\right| \int_{-\tau}^{0} e^{-\lambda_{0} s} d s\right\}\|\phi\| .
\end{aligned}
$$

By (54), we have

$$
\left|L\left(\lambda_{0} ; \phi\right)\right| \leq k_{\lambda_{0}}\|\phi\|_{C^{2}} .
$$

Consider the function $\Phi_{1}\left(\lambda_{0} ; \phi\right)$ defined by (8). Then, by (59), we have

$$
\left\|\Phi_{1}\left(\lambda_{0} ; \phi\right)\right\| \leq m\left(\lambda_{0}, \delta_{0}, \gamma_{0}\right)\|\phi\|+\frac{\left|L\left(\lambda_{0} ; \phi\right)\right|}{\left|\beta_{\lambda_{0}}\right|}
$$

and so, in view of (63),

$$
\left\|\Phi_{1}\left(\lambda_{0} ; \phi\right)\right\| \leq m\left(\lambda_{0}, \delta_{0}, \gamma_{0}\right)\|\phi\|+\frac{k_{\lambda_{0}}}{\left|\beta_{\lambda_{0}}\right|}\|\phi\|_{C^{2}}
$$


Therefore,

$$
\left\|\Phi_{1}\left(\lambda_{0} ; \phi\right)\right\| \leq\left(m\left(\lambda_{0}, \delta_{0}, \gamma_{0}\right)+\frac{k_{\lambda_{0}}}{\left|\beta_{\lambda_{0}}\right|}\right)\|\phi\|_{C^{2}}
$$

Furthermore,

$$
\left\|\left(\Phi_{1}\left(\lambda_{0} ; \phi\right)\right)^{\prime}\right\| \leq m\left(\lambda_{0}, \delta_{0}, \gamma_{0}\right)\left(\left\|\phi^{\prime}\right\|+\left|\lambda_{0}\right|\|\phi\|\right)
$$

where $\left(\Phi_{1}\left(\lambda_{0} ; \phi\right)\right)^{\prime}$ is derivative of $\Phi_{1}\left(\lambda_{0} ; \phi\right)$, and therefore,

$$
\left\|\left(\Phi_{1}\left(\lambda_{0} ; \phi\right)\right)^{\prime}\right\| \leq m\left(\lambda_{0}, \delta_{0}, \gamma_{0}\right)\left(1+\left|\lambda_{0}\right|\right)\|\phi\|_{C^{2}} .
$$

Let us consider the constant $R\left(\lambda_{0}, \delta_{0} ; \phi\right)$ defined by (20). Then, by (64) and (65), we have

$$
\begin{aligned}
\left|R\left(\lambda_{0}, \delta_{0} ; \phi\right)\right| \leq & \left|\left(\Phi_{1}\left(\lambda_{0} ; \phi\right)\right)^{\prime}(0)\right|+\left|\delta_{0}\right|\left|\Phi_{1}\left(\lambda_{0} ; \phi\right)(0)\right|+\left|p_{1}-3 \lambda_{0}-2 \delta_{0}\right|\left|\Phi_{1}\left(\lambda_{0} ; \phi\right)(0)\right| \\
& +e^{-\lambda_{0} \tau}\left|p_{2}\right|\left|\Phi_{1}\left(\lambda_{0} ; \phi\right)(-\tau)\right|+e^{-\left(\lambda_{0}+\delta_{0}\right) \tau}\left|p_{2} \delta_{0}+q_{2}+2 p_{2} \lambda_{0}\right| \int_{-\tau}^{0} e^{-\delta_{0} s}\left|\Phi_{1}\left(\lambda_{0} ; \phi\right)(s)\right| d s \\
\leq & \left|\left(\Phi_{1}\left(\lambda_{0} ; \phi\right)\right)^{\prime}\right||+| \delta_{0}\left|\left\|\Phi_{1}\left(\lambda_{0} ; \phi\right)\right\|+\right| p_{1}-3 \lambda_{0}-2 \delta_{0}\left|\left\|\Phi_{1}\left(\lambda_{0} ; \phi\right)\right\|+e^{-\lambda_{0} \tau}\right| p_{2} \mid\left\|\Phi_{1}\left(\lambda_{0} ; \phi\right)\right\| \\
& +e^{-\left(\lambda_{0}+\delta_{0}\right) \tau}\left|p_{2} \delta_{0}+q_{2}+2 p_{2} \lambda_{0}\right| \int_{-\tau}^{0} e^{-\delta_{0} s}\left\|\Phi_{1}\left(\lambda_{0} ; \phi\right)\right\| d s \\
\leq & \left\{m\left(\lambda_{0}, \delta_{0}, \gamma_{0}\right)\left(1+\left|\lambda_{0}\right|\right)+\left|\delta_{0}\right|\left(m\left(\lambda_{0}, \delta_{0}, \gamma_{0}\right)+\frac{k_{\lambda_{0}}}{\left|\beta_{\lambda_{0}}\right|}\right)+e^{-\lambda_{0} \tau}\left|p_{2}\right|\left(m\left(\lambda_{0}\right)+\frac{k_{\lambda_{0}}}{\left|\beta_{\lambda_{0}}\right|}\right)\right. \\
& \left.+e^{-\left(\lambda_{0}+\delta_{0}\right) \tau}\left|p_{2} \delta_{0}+q_{2}+2 p_{2} \lambda_{0}\right| \int_{-\tau}^{0} e^{-\delta_{0} s}\left(m\left(\lambda_{0}, \delta_{0}, \gamma_{0}\right)+\frac{k_{\lambda_{0}}}{\left|\beta_{\lambda_{0}}\right|}\right) d s\right\}\|\phi\|_{C^{2}} \\
\leq & m\left(\lambda_{0}, \delta_{0}, \gamma_{0}\right)\left\{1+\left|\lambda_{0}\right|+\left|\delta_{0}\right|\left(1+\frac{k_{\lambda_{0}}}{\left|\beta_{\lambda_{0}}\right|}\right)+\left|p_{1}-3 \lambda_{0}-2 \delta_{0}\right|\left(1+\frac{k_{\lambda_{0}}}{\left|\beta_{\lambda_{0}}\right|}\right)+e^{-\lambda_{0} \tau}\left|p_{2}\right|\left(1+\frac{k_{\lambda_{0}}}{\left|\beta_{\lambda_{0}}\right|}\right)\right. \\
& \left.+e^{-\left(\lambda_{0}+\delta_{0}\right) \tau}\left|p_{2} \delta_{0}+q_{2}+2 p_{2} \lambda_{0}\right| \int_{-\tau}^{0} e^{-\delta_{0} s}\left(1+\frac{k_{\lambda_{0}}}{\left|\beta_{\lambda_{0}}\right|}\right) d s\right\}\|\phi\|_{C^{2}} \\
\leq & m\left(\lambda_{0}, \delta_{0}, \gamma_{0}\right)\left(1+\frac{k_{\lambda_{0}}}{\left|\beta_{\lambda_{0}}\right|}\right)\left\{\left(1+\left|\lambda_{0}\right|+\left|\delta_{0}\right|\right)+\left|p_{1}-3 \lambda_{0}-2 \delta_{0}\right|+e^{-\lambda_{0} \tau}\left|p_{2}\right|\right. \\
& \left.+e^{-\left(\lambda_{0}+\delta_{0}\right) \tau}\left|p_{2} \delta_{0}+q_{2}+2 p_{2} \lambda_{0}\right| \int_{-\tau}^{0} e^{-\delta_{0} s} d s\right\}\|\phi\|_{C^{2}} .
\end{aligned}
$$

By (52) and (55), we have

$$
\left|R\left(\lambda_{0}, \delta_{0} ; \phi\right)\right| \leq m\left(\lambda_{0}, \delta_{0}, \gamma_{0}\right) h_{\lambda_{0}, \delta_{0}}\|\phi\|_{C^{2}} .
$$

Consider the function $\Phi_{2}\left(\lambda_{0}, \delta_{0} ; \phi\right)$ defined by (21). Then, by (8), (60) and (62), we have

$$
\left\|\Phi_{2}\left(\lambda_{0}, \delta_{0} ; \phi\right)\right\| \leq m\left(\lambda_{0}, \delta_{0}, \gamma_{0}\right)\|\phi\|+\frac{\left|L\left(\lambda_{0} ; \phi\right)\right|}{\left|\beta_{\lambda_{0}}\right|} m\left(\lambda_{0}, \delta_{0}, \gamma_{0}\right)+\frac{\left|R\left(\lambda_{0}, \delta_{0} ; \phi\right)\right|}{\left|\eta_{\lambda_{0}, \delta_{0}}\right|}
$$

and so, in view of (63) and (66),

$$
\left\|\Phi_{2}\left(\lambda_{0}, \delta_{0} ; \phi\right)\right\| \leq m\left(\lambda_{0}, \delta_{0}, \gamma_{0}\right)\|\phi\|_{C^{2}}+m\left(\lambda_{0}, \delta_{0}, \gamma_{0}\right) \frac{k_{\lambda_{0}}}{\left|\beta_{\lambda_{0}}\right|}\|\phi\|_{C^{2}}+\frac{h_{\lambda_{0}, \delta_{0}}}{\left|\eta_{\lambda_{0}, \delta_{0}}\right|}\|\phi\|_{C^{2}}
$$


Therefore,

$$
\left\|\Phi_{2}\left(\lambda_{0}, \delta_{0} ; \phi\right)\right\| \leq m\left(\lambda_{0}, \delta_{0}, \gamma_{0}\right)\left\{1+\frac{k_{\lambda_{0}}}{\left|\beta_{\lambda_{0}}\right|}+\frac{h_{\lambda_{0}, \delta_{0}}}{\left|\eta_{\lambda_{0}, \delta_{0}}\right|}\right\}\|\phi\|_{C^{2}} \equiv m\left(\lambda_{0}, \delta_{0}, \gamma_{0}\right) d_{\lambda_{0}, \delta_{0}}\|\phi\|_{C^{2}}
$$

Let us consider the constant $K\left(\lambda_{0}, \delta_{0}, \gamma_{0} ; \phi\right)$ defined by (31). Then, by (67), we have

$$
\begin{aligned}
\left|K\left(\lambda_{0}, \delta_{0}, \gamma_{0} ; \phi\right)\right| \leq & \left|\Phi_{2}\left(\lambda_{0}, \delta_{0} ; \phi\right)(0)\right|+e^{-\left(\lambda_{0}+\delta_{0}+\gamma_{0}\right) \tau}\left|p_{2}\right| \int_{-\tau}^{0} e^{-\gamma_{0} s}\left|\Phi_{2}\left(\lambda_{0}, \delta_{0} ; \phi\right)(s)\right| d s \\
& +e^{-\left(\lambda_{0}+\delta_{0}\right) \tau}\left|p_{2} \delta_{0}+q_{2}+2 p_{2} \lambda_{0}\right| \int_{0}^{\tau} e^{-\gamma_{0} s}\left\{\int_{-s}^{0} e^{-\gamma_{0} u}\left|\Phi_{2}\left(\lambda_{0}, \delta_{0} ; \phi\right)(u)\right| d u\right\} d s \\
& +e^{-\lambda_{0} \tau}\left|p_{2} \lambda_{0}^{2}+q_{2} \lambda_{0}+v_{2}\right| \int_{0}^{\tau} e^{-\delta_{0} s}\left\{\int_{0}^{s} e^{-\gamma_{0} u}\left\{\int_{-u}^{0} e^{-\gamma_{0} \omega}\left|\Phi_{2}\left(\lambda_{0}, \delta_{0} ; \phi\right)(\omega)\right| d \omega\right\} d u\right\} d s \\
\leq & \left|\Phi_{2}\left(\lambda_{0}, \delta_{0} ; \phi\right)\left\|+e^{-\left(\lambda_{0}+\delta_{0}+\gamma_{0}\right) \tau}\left|p_{2}\right| \int_{-\tau}^{0} e^{-\gamma_{0} s}\right\| \Phi_{2}\left(\lambda_{0}, \delta_{0} ; \phi\right) \| d s\right. \\
& +e^{-\left(\lambda_{0}+\delta_{0}\right) \tau}\left|p_{2} \delta_{0}+q_{2}+2 p_{2} \lambda_{0}\right| \int_{0}^{\tau} e^{-\gamma_{0} s}\left\{\int_{-s}^{0} e^{-\gamma_{0} u}\left\|\Phi_{2}\left(\lambda_{0}, \delta_{0} ; \phi\right)\right\| d u\right\} d s \\
& +e^{-\lambda_{0} \tau}\left|p_{2} \lambda_{0}^{2}+q_{2} \lambda_{0}+v_{2}\right| \int_{0}^{\tau} e^{-\delta_{0} s}\left\{\int_{0}^{s} e^{-\gamma_{0} u}\left\{\int_{-u}^{0} e^{-\gamma_{0} \omega}\left\|\Phi_{2}\left(\lambda_{0}, \delta_{0} ; \phi\right)\right\| d \omega\right\} d u\right\} d s \\
\leq & m\left(\lambda_{0}, \delta_{0}, \gamma_{0}\right)\left\{1+e^{-\left(\lambda_{0}+\delta_{0}+\gamma_{0}\right) \tau}\left|p_{2}\right| \int_{-\tau}^{0} e^{-\gamma_{0} s} d s\right. \\
& +e^{-\left(\lambda_{0}+\delta_{0}\right) \tau}\left|p_{2} \delta_{0}+q_{2}+2 p_{2} \lambda_{0}\right| \int_{0}^{\tau} e^{-\gamma_{0} s}\left\{\int_{-s}^{0} e^{-\gamma_{0} u} d u\right\} d s \\
& \left.e^{-\lambda_{0} \tau}\left|p_{2} \lambda_{0}^{2}+q_{2} \lambda_{0}+v_{2}\right| \int_{0}^{\tau} e^{-\delta_{0} s}\left\{\int_{0}^{s} e^{-\gamma_{0} u}\left\{\int_{-u}^{0} e^{-\gamma_{0} \omega} d \omega\right\} d u\right\} d s\right\} d \lambda_{0}, \delta_{0}\|\phi\|_{C^{2}} .
\end{aligned}
$$

By (58), we have

$$
\left|K\left(\lambda_{0}, \delta_{0}, \gamma_{0} ; \phi\right)\right| \leq m\left(\lambda_{0}, \delta_{0}, \gamma_{0}\right) \ell_{\lambda_{0}, \delta_{0}, \gamma_{0}} d_{\lambda_{0}, \delta_{0}}\|\phi\|_{C^{2}}
$$

Let $\xi_{\lambda_{0}, \delta_{0}, \gamma_{0}}$ be defined by (30), ( Note that, because of $\mu_{\lambda_{0}, \delta_{0}, \gamma_{0}}<1$ by (32), we always have $1+\xi_{\lambda_{0}, \delta_{0}, \gamma_{0}}>0$.) and define $M\left(\lambda_{0}, \delta_{0}, \gamma_{0} ; \phi\right)$ by (44). Then, by using (61), we have

$$
M\left(\lambda_{0}, \delta_{0}, \gamma_{0} ; \phi\right) \leq m\left(\lambda_{0}, \delta_{0}, \gamma_{0}\right)\left\|\Phi_{2}\left(\lambda_{0}, \delta_{0} ; \phi\right)\right\|+\frac{\left|K\left(\lambda_{0}, \delta_{0}, \gamma_{0} ; \phi\right)\right|}{1+\xi_{\lambda_{0}, \delta_{0}, \gamma_{0}}}
$$

So, by virtue of (67) and (68),

$$
M\left(\lambda_{0}, \delta_{0}, \gamma_{0} ; \phi\right) \leq m\left(\lambda_{0}, \delta_{0}, \gamma_{0}\right)\left(m\left(\lambda_{0}, \delta_{0}, \gamma_{0}\right)+\frac{\ell_{\lambda_{0}, \delta_{0}, \gamma_{0}}}{1+\xi_{\lambda_{0}, \delta_{0}, \gamma_{0}}}\right) d_{\lambda_{0}, \delta_{0}}\|\phi\|_{C^{2}}
$$

Now, let $y$ be the solution of the IVP (1) and (2), and define the functions $z$ by (9) and $w$ by (22). Also, we define the functions $\Omega$ and $\Theta$ by (38) and (42), respectively. Note that (30) (which is a consequence of the assumption (32) ) states that $1+\xi_{\lambda_{0}, \delta_{0}, \gamma_{0}}>0$. Then, as in the proof of the Theorem 1, we show that (48) and (51) are satisfied. We shall prove that $y$ satisfies (53), where the constants $m\left(\lambda_{0}, \delta_{0,}, \gamma_{0}\right), k_{\lambda_{0}}, h_{\lambda_{0}, \delta_{0}}, d_{\lambda_{0}, \delta_{0}}, g\left(\lambda_{0}, \delta_{0}, \gamma_{0}\right)$ and $\ell_{\lambda_{0}, \delta_{0}, \gamma_{0}}$ are defined by (52), (54), (55), (56), (57) and (58), respectively. 
From (51) it follows that

$$
y(t)=\frac{L\left(\lambda_{0} ; \phi\right)}{\beta_{\lambda_{0}}} e^{\lambda_{0} t}+\frac{R\left(\lambda_{0}, \delta_{0} ; \phi\right)}{\eta_{\lambda_{0}, \delta_{0}}} e^{\left(\lambda_{0}+\delta_{0}\right) t}+\left[\Theta(t)+\frac{K\left(\lambda_{0}, \delta_{0}, \gamma_{0} ; \phi\right)}{1+\xi_{\lambda_{0}, \delta_{0}, \gamma_{0}}}\right] e^{\left(\lambda_{0}+\delta_{0}+\gamma_{0}\right) t}
$$

for $t \geq 0$ and consequently

$$
|y(t)| \leq \frac{\left|L\left(\lambda_{0} ; \phi\right)\right|}{\left|\beta_{\lambda_{0}}\right|} e^{\lambda_{0} t}+\frac{\left|R\left(\lambda_{0}, \delta_{0} ; \phi\right)\right|}{\left|\eta_{\lambda_{0}, \delta_{0}}\right|} e^{\left(\lambda_{0}+\delta_{0}\right) t}+\left[|\Theta(t)|+\frac{\left|K\left(\lambda_{0}, \delta_{0}, \gamma_{0} ; \phi\right)\right|}{1+\xi_{\lambda_{0}, \delta_{0}, \gamma_{0}}}\right] e^{\left(\lambda_{0}+\delta_{0}+\gamma_{0}\right) t} .
$$

Thus, using (48), we obtain

$$
|y(t)| \leq \frac{\left|L\left(\lambda_{0} ; \phi\right)\right|}{\left|\beta_{\lambda_{0}}\right|} e^{\lambda_{0} t}+\frac{\left|R\left(\lambda_{0}, \delta_{0} ; \phi\right)\right|}{\left|\eta_{\lambda_{0}, \delta_{0}}\right|} e^{\left(\lambda_{0}+\delta_{0}\right) t}+\left[\mu_{\lambda_{0}, \delta_{0}, \gamma_{0}} M\left(\lambda_{0}, \delta_{0}, \gamma_{0} ; \phi\right)+\frac{\left|K\left(\lambda_{0}, \delta_{0}, \gamma_{0} ; \phi\right)\right|}{1+\xi_{\lambda_{0}, \delta_{0}, \gamma_{0}}}\right] e^{\left(\lambda_{0}+\delta_{0}+\gamma_{0}\right) t}
$$

for $t \geq 0$. Using (63) and (66), we obtain

$$
\frac{\left|L\left(\lambda_{0} ; \phi\right)\right|}{\left|\beta_{\lambda_{0}}\right|} \leq \frac{k_{\lambda_{0}}}{\left|\beta_{\lambda_{0}}\right|}\|\phi\|_{C^{2}}
$$

and

$$
\frac{\left|R\left(\lambda_{0}, \delta_{0} ; \phi\right)\right|}{\left|\eta_{\lambda_{0}, \delta_{0}}\right|} \leq \frac{m\left(\lambda_{0}, \delta_{0}, \gamma_{0}\right) h_{\lambda_{0}, \delta_{0}}}{\left|\eta_{\lambda_{0}, \delta_{0}}\right|}\|\phi\|_{C^{2}}
$$

Moreover, by the use of (68) and (69), we get

$$
\begin{aligned}
\mu_{\lambda_{0}, \delta_{0}, \gamma_{0}} M\left(\lambda_{0}, \delta_{0}, \gamma_{0} ; \phi\right)+\frac{\left|K\left(\lambda_{0}, \delta_{0}, \gamma_{0} ; \phi\right)\right|}{1+\xi_{\lambda_{0}, \delta_{0}, \gamma_{0}}} \leq & \mu_{\lambda_{0}, \delta_{0}, \gamma_{0}} m\left(\lambda_{0}, \delta_{0}, \gamma_{0}\right)\left(m\left(\lambda_{0}, \delta_{0}, \gamma_{0}\right)+\frac{\ell_{\lambda_{0}, \delta_{0}, \gamma_{0}}}{1+\xi_{\lambda_{0}, \delta_{0}, \gamma_{0}}}\right) d_{\lambda_{0}, \delta_{0}}\|\phi\|_{C^{2}} \\
& +\frac{m\left(\lambda_{0}, \delta_{0}, \gamma_{0}\right) \ell_{\lambda_{0}, \delta_{0}, \gamma_{0}} d_{\lambda_{0}, \delta_{0}}}{1+\xi_{\lambda_{0}, \delta_{0}, \gamma_{0}}}\|\phi\|_{C^{2}}
\end{aligned}
$$

So, because of (57), we have

$$
\mu_{\lambda_{0}, \delta_{0}, \gamma_{0}} M\left(\lambda_{0}, \delta_{0}, \gamma_{0} ; \phi\right)+\frac{\left|K\left(\lambda_{0}, \delta_{0}, \gamma_{0} ; \phi\right)\right|}{1+\xi_{\lambda_{0}, \delta_{0}, \gamma_{0}}} \leq m\left(\lambda_{0}, \delta_{0}, \gamma_{0}\right) d_{\lambda_{0}, \delta_{0}} g\left(\lambda_{0}, \delta_{0,}, \gamma_{0}\right)\|\phi\|_{C^{2}}
$$

Using (71), (72) and (73), we immediately see that (70) implies (53). Hence, (53) has been proved.

Finally, we will establish that the constant $g\left(\lambda_{0}, \delta_{0}, \gamma_{0}\right)$ is greater than 1 . By (35) and since $1+\xi_{\lambda_{0}, \delta_{0}, \gamma_{0}}>0$, we have

$$
0<1+\xi_{\lambda_{0}, \delta_{0}, \gamma_{0}} \leq 1+\left|\xi_{\lambda_{0}, \delta_{0}, \gamma_{0}}\right| \leq 1+\mu_{\lambda_{0}, \delta_{0}, \gamma_{0}}
$$

which ensures that

$$
1 \leq \frac{1+\mu_{\lambda_{0}, \delta_{0}, \gamma_{0}}}{1+\xi_{\lambda_{0}, \delta_{0}, \gamma_{0}}}
$$

Furthermore, from (58) we have

$$
\ell_{\lambda_{0}, \delta_{0}, \gamma_{0}}>1
$$

Thus, we obtain

$$
1<\frac{1+\mu_{\lambda_{0}, \delta_{0}, \gamma_{0}}}{1+\xi_{\lambda_{0}, \delta_{0}, \gamma_{0}}} \ell_{\lambda_{0}, \delta_{0}, \gamma_{0}}
$$


Hence, it follows from the definition of $g\left(\lambda_{0}, \delta_{0}, \gamma_{0}\right)$ by (57) that $g\left(\lambda_{0}, \delta_{0}, \gamma_{0}\right)$ is always greater than 1 . The proof of the theorem is now complete.

Proof. (Proof of the Corollary 1.) Let $y$ be the solution of the IVP (1) and (2). By Theorem 1, the solution $y$ satisfies (53), where $\beta_{\lambda_{0}}, \eta_{\lambda_{0}, \delta_{0}}, m\left(\lambda_{0}, \delta_{0}, \gamma_{0}\right), k_{\lambda_{0}}, h_{\lambda_{0}, \delta_{0}}, d_{\lambda_{0}, \delta_{0}}$ and $g\left(\lambda_{0}, \delta_{0}, \gamma_{0}\right)$, are defined by (6), (19), (52), (54), (55), (56) and (57), respectively. The constant $g\left(\lambda_{0}, \delta_{0}, \gamma_{0}\right)$ is greater than 1 .

Assume first that $\lambda_{0} \leq 0, \lambda_{0}+\delta_{0} \leq 0$ and $\lambda_{0}+\delta_{0}+\gamma_{0} \leq 0$. Then (53), gives

$$
|y(t)| \leq\left\{\frac{k_{\lambda_{0}}}{\left|\beta_{\lambda_{0}}\right|}+\frac{m\left(\lambda_{0}, \delta_{0}, \gamma_{0}\right) h_{\lambda_{0}, \delta_{0}}}{\left|\eta_{\lambda_{0}, \delta_{0}}\right|}+m\left(\lambda_{0}, \delta_{0}, \gamma_{0}\right) d_{\lambda_{0}, \delta_{0}} g\left(\lambda_{0}, \delta_{0}, \gamma_{0}\right)\right\}\|\phi\|_{C^{2}} \quad \text { for } \quad t \geq 0 .
$$

So, if we set

$$
S\left(\lambda_{0}, \delta_{0}, \gamma_{0}\right)=\frac{k_{\lambda_{0}}}{\left|\beta_{\lambda_{0}}\right|}+\frac{m\left(\lambda_{0}, \delta_{0}, \gamma_{0}\right) h_{\lambda_{0}, \delta_{0}}}{\left|\eta_{\lambda_{0}, \delta_{0}}\right|}+m\left(\lambda_{0}, \delta_{0}, \gamma_{0}\right) d_{\lambda_{0}, \delta_{0}} g\left(\lambda_{0}, \delta_{0}, \gamma_{0}\right)
$$

then we have

$$
|y(t)| \leq S\left(\lambda_{0}, \delta_{0}, \gamma_{0}\right)\|\phi\|_{C^{2}} \quad \text { for every } \quad t \geq 0 .
$$

Since $m\left(\lambda_{0}, \delta_{0}, \gamma_{0}\right)>1, d_{\lambda_{0}, \delta_{0}}>1$ and $g\left(\lambda_{0}, \delta_{0,} \gamma_{0}\right)>1$, we always have $S\left(\lambda_{0}, \delta_{0,}, \gamma_{0}\right)>1$. Thus, we obtain

$$
|y(t)| \leq S\left(\lambda_{0}, \delta_{0}, \gamma_{0}\right)\|\phi\|_{C^{2}} \text { for all } t \geq-\tau
$$

Using this inequality, we can immediately verify that the trivial solution of (1) is stable (at 0 ).

Next, let us suppose that $\lambda_{0}<0, \lambda_{0}+\delta_{0}<0$ and $\lambda_{0}+\delta_{0}+\gamma_{0}<0$. Then the trivial solution of (1) is stable (at 0 ).

Furthermore, we see that it follows from (53) that the solution $y$ satisfies

$$
\lim _{t \rightarrow \infty} y(t)=0
$$

Hence, the trivial solution of (1) is asymptotically stable (at 0 ). The proof of Corollary 1 is completed.

\section{Examples}

Example 1. Consider

$$
\begin{aligned}
y^{\prime \prime \prime}(t) & =\left(\frac{e-8}{2}\right)\left(y^{\prime \prime}(t)+y^{\prime}(t)\right)+\frac{1}{2 e^{2}}\left(y^{\prime \prime}(t-1)-y^{\prime}(t-1)\right)+\left(\frac{1}{e-1}\right)(y(t)-y(t-1)), t \geq 0 \\
y(t) & =\phi(t),-1 \leq t \leq 0,
\end{aligned}
$$

where $\phi(t)$ is an arbitrary twice continuously differentiable initial function on the interval $[-1,0]$. In this example we apply the characteristic equations (3), (5) and (18). That is, the characteristic equation (3) is

$$
\lambda^{3}=\left(\lambda^{2}+\lambda\right)\left(\frac{e-8}{2}+\frac{e^{-\lambda}}{2 e^{2}}\right)+\left(\frac{1}{e-1}\right)\left(1-e^{-\lambda}\right)
$$


and we see that $\lambda=0, \lambda=-1$ and $\lambda=-2$ are real roots of (75). Let $\lambda=0$. Then, for $\lambda_{0}=0$ the characteristic equation (5) is

$$
\delta^{2}=(\delta+1)\left(\frac{e-8}{2}+\frac{e^{-\delta}}{2 e^{2}}\right)+\left(\frac{1}{e-1}\right)\left(\frac{1-e^{-\delta}}{\delta}\right) .
$$

Therefore, $\delta=-1, \delta=-2$ are real roots of (76). Let $\delta=-1$. Then, for $\lambda_{0}=0$ and $\delta_{0}=-1$ the characteristic equation (18) is

$$
\gamma=\frac{e-4}{2}+\frac{e^{1-\gamma}}{2 e^{2}}-\frac{1}{\gamma}+\frac{1}{(e-1)} \frac{1-e^{1-\gamma}}{\gamma(\gamma-1)},
$$

and we see that $\gamma=\gamma_{0}=-1$ is a real root of (77) and the conditions of Corollary 1 are satisfied. That is,

$$
\delta_{0}=-1 \neq 0, \gamma_{0}=-1 \neq 0, \gamma_{0} \neq-\delta_{0}
$$

and

$$
\mu_{\lambda_{0}, \delta_{0}, \gamma_{0}}=\mu_{0,-1,-1} \cong 0.86142<1 \text {. }
$$

Since $\lambda_{0}=0, \lambda_{0}+\delta_{0}=-1<0$ and $\lambda_{0}+\delta_{0}+\gamma_{0}=-2<0$ the zero solution of (74) is stable.

\section{Example 2. Consider}

$$
\begin{aligned}
y^{\prime \prime \prime}(t) & =-13 y^{\prime \prime}(t)+\frac{1}{e^{3}} y^{\prime \prime}(t-0.5)-50 y^{\prime}(t)+\frac{6}{e^{3}} y^{\prime}(t-0.5)-56 y(t)+\frac{8}{e^{3}} y(t-0.5), t \geq 0, \\
y(t) & =\phi(t),-0.5 \leq t \leq 0
\end{aligned}
$$

where $\phi(t)$ is an arbitrary twice continuously differentiable initial function on the interval $[-0.5,0]$. In this example we apply the characteristic equations (3), (5) and (18). That is, the characteristic equation (3) is

$$
\lambda^{3}=-13 \lambda^{2}-50 \lambda-56+\frac{e^{-\frac{\lambda}{2}}}{e^{3}}\left(\lambda^{2}+6 \lambda+8\right),
$$

and we see $\lambda=-2, \lambda=-4$ and $\lambda=-6$ are real roots of (78). Let $\lambda=-2$. Then, for $\lambda_{0}=-2$ the characteristic equation (5) is

$$
\delta^{2}=-7 \delta-10+(\delta+2) e^{-\frac{(\delta+4)}{2}}
$$

Therefore, we see that $\delta=-2$ and $\delta=-4$ are roots of (79). Let $\delta=-2$. Then, for $\lambda_{0}=-2$ and $\delta_{0}=-2$ the characteristic equation (18) is

$$
\gamma=-3+e^{-\frac{\gamma+2}{2}}
$$

and we find that $\gamma=\gamma_{0}=-2$ is a real root of (80). Corresponding to the roots $\lambda_{0}=-2, \delta_{0}=-2$ and $\gamma_{0}=-2$, the conditions of Corollary 1 are satisfied. That is,

$$
\begin{gathered}
\delta_{0}=-2 \neq 0, \gamma_{0}=-2 \neq 0, \gamma_{0} \neq-\delta_{0} \\
\mu_{\lambda_{0}, \delta_{0}, \gamma_{0}}=\mu_{-2,-2,-2}=\frac{1}{2}<1 .
\end{gathered}
$$

Since $\lambda_{0}=-2<0, \lambda_{0}+\delta_{0}=-4<0$ and $\lambda_{0}+\delta_{0}+\gamma_{0}=-6<0$ the zero solution of the given equation is asymptotically stable. 


\section{A result on the behavior of the solutions}

We begin this section with the following lemma.

Lemma 1. Suppose that

$$
p_{2}<0,\left(p_{2} \delta_{0}+q_{2}+2 p_{2} \lambda_{0}\right)>0 \text { and }\left(p_{2} \lambda_{0}^{2}+q_{2} \lambda_{0}+v_{2}\right)<0
$$

Let $\lambda_{0}, \delta_{0}$ and $\gamma_{0}$ be real roots of the characteristic equations (3), (5) and (18), respectively, and define $\xi_{\lambda_{0}, \delta_{0}, \gamma_{0}}$ by (30). Then $1+\xi_{\lambda_{0}, \delta_{0}, \gamma_{0}}>0$ if (18) has another real root less than $\gamma_{0}$, and $1+\xi_{\lambda_{0}, \delta_{0}, \gamma_{0}}<0$ if (18) has another real root greater than $\gamma_{0}$.

Proof. Let $G(\gamma)$ denote the characteristic function of (18), i.e.,

$$
\begin{aligned}
G(\gamma)= & \gamma-\left(p_{1}-3 \lambda_{0}-2 \delta_{0}\right)-p_{2} e^{-\left(\lambda_{0}+\delta_{0}+\gamma\right) \tau}+e^{-\left(\lambda_{0}+\delta_{0}\right) \tau}\left(p_{2} \delta_{0}+q_{2}+2 p_{2} \lambda_{0}\right) \gamma^{-1}\left(1-e^{-\gamma \tau}\right) \\
& -e^{-\lambda_{0} \tau}\left(p_{2} \lambda_{0}^{2}+q_{2} \lambda_{0}+v_{2}\right) \gamma^{-1}\left\{\delta_{0}^{-1}\left(1-e^{-\delta_{0} \tau}\right)-\left(\delta_{0}+\gamma\right)^{-1}\left(1-e^{-\left(\delta_{0}+\gamma\right) \tau}\right)\right\}
\end{aligned}
$$

or

$$
\begin{aligned}
G(\gamma)= & \gamma-\left(p_{1}-3 \lambda_{0}-2 \delta_{0}\right)-p_{2} e^{-\left(\lambda_{0}+\delta_{0}+\gamma\right) \tau}+e^{-\left(\lambda_{0}+\delta_{0}\right) \tau}\left(p_{2} \delta_{0}+q_{2}+2 p_{2} \lambda_{0}\right) \int_{0}^{\tau} e^{-\gamma_{s}} d s \\
& -e^{-\lambda_{0} \tau}\left(p_{2} \lambda_{0}^{2}+q_{2} \lambda_{0}+v_{2}\right) \int_{0}^{\tau} e^{-\delta_{0} s}\left(\int_{0}^{s} e^{-\gamma u} d u\right) d s
\end{aligned}
$$

for $\gamma \in \mathbb{R}$. We obtain immediately

$$
\begin{aligned}
G^{\prime}(\gamma)= & +p_{2} \tau e^{-\left(\lambda_{0}+\delta_{0}+\gamma\right) \tau}-e^{-\left(\lambda_{0}+\delta_{0}\right) \tau}\left(p_{2} \delta_{0}+q_{2}+2 p_{2} \lambda_{0}\right) \int_{0}^{\tau} s e^{-\gamma_{s}} d s \\
& +e^{-\lambda_{0} \tau}\left(p_{2} \lambda_{0}^{2}+q_{2} \lambda_{0}+v_{2}\right) \int_{0}^{\tau} e^{-\delta_{0} s}\left(\int_{0}^{s} u e^{-\gamma u} d u\right) d s
\end{aligned}
$$

for $\gamma \in \mathbb{R}$. Furthermore,

$$
\begin{aligned}
G^{\prime \prime}(\gamma)= & -p_{2} \tau^{2} e^{-\left(\lambda_{0}+\delta_{0}+\gamma\right) \tau}+e^{-\left(\lambda_{0}+\delta_{0}\right) \tau}\left(p_{2} \delta_{0}+q_{2}+2 p_{2} \lambda_{0}\right) \int_{0}^{\tau} s^{2} e^{-\gamma s} d s \\
& -e^{-\lambda_{0} \tau}\left(p_{2} \lambda_{0}^{2}+q_{2} \lambda_{0}+v_{2}\right) \int_{0}^{\tau} e^{-\delta_{0} s}\left(\int_{0}^{s} u^{2} e^{-\gamma u} d u\right) d s
\end{aligned}
$$

for $\gamma \in \mathbb{R}$. So, taking into account (81), we conclude that

$$
G^{\prime \prime}(\gamma)>0 \text { for } \gamma \in \mathbb{R}
$$

Now, assume that (18) has another real root $\gamma_{1}$ with $\gamma_{1}<\gamma_{0}$ (respectively, $\gamma_{1}>\gamma_{0}$ ). From the definition of the function $G$ by (82) it follows that $G\left(\gamma_{1}\right)=G\left(\gamma_{0}\right)=0$, and consequently Rolle's Theorem guarantees the existence of a point $\alpha$ with $\gamma_{1}<\alpha<\gamma_{0}$ (resp., $\gamma_{1}>\alpha>\gamma_{0}$ ) such that $G^{\prime}(\alpha)=0$. But, (84) implies that $G^{\prime}$ is positive on $(\alpha, \infty)$ (resp., $G^{\prime}$ is negative on $(-\infty, \alpha)$ ). Thus we must have $G^{\prime}\left(\gamma_{0}\right)>0$ (resp., $\left.G^{\prime}\left(\gamma_{0}\right)<0\right)$. By taking into account the definition of $\xi_{\lambda_{0}, \delta_{0}, \gamma_{0}}$ by (30), from (83) we obtain

$$
G^{\prime}\left(\gamma_{0}\right)=1+\xi_{\lambda_{0}, \delta_{0}, \gamma_{0}}
$$

and so the proof of the lemma is complete. 
Now, we will establish the following theorem.

Theorem 3. Suppose that statement (81) is true. Let $\lambda_{0}$ be real root of the characteristic equation (3), and let $\beta_{\lambda_{0}}$ and $L\left(\lambda_{0} ; \phi\right)$ be defined by (6) and (7), respectively. Furthermore, let $\delta_{0}$ be real root of the characteristic equation (5) and let $\eta_{\lambda_{0}, a_{0}}$ and $R\left(\lambda_{0}, \delta_{0} ; \phi\right)$ be defined (19) and (20), respectively. Suppose that $\beta_{\lambda_{0}} \neq 0$ and $\eta_{\lambda_{0}, \delta_{0}} \neq 0$. Moreover, let $\gamma_{0}$ be a real root of the characteristic equation (18), and let $\xi_{\lambda_{0}, \delta_{0}, \gamma_{0}}$ and $K\left(\lambda_{0}, \delta_{0}, \gamma_{0} ; \phi\right)$ be defined by (30) and (31), respectively. Also, let $\gamma_{1}$ be real root of (18) with $\gamma_{1} \neq \gamma_{0}$. (Note that, because of $\beta_{\lambda_{0}} \neq 0$, we have $\delta_{0} \neq 0$ and $\gamma_{0} \neq-\delta_{0}$, $\gamma_{1} \neq-\delta_{0}$. Furthermore, because of $\eta_{\lambda_{0}, \delta_{0}} \neq 0$, we have $\gamma_{0} \neq 0$ and $\gamma_{1} \neq 0$. Moreover, note that Lemma 1. guarantees that $1+\xi_{\lambda_{0}, \delta_{0}, \gamma_{0}} \neq 0$.

Then the solution $y$ of the IVP (1) and (2) satisfies

$$
\begin{aligned}
C_{1}\left(\lambda_{0}, \delta_{0}, \gamma_{0}, \gamma_{1} ; \phi\right) & \leq e^{-\gamma_{1} t}\left[e^{-\left(\lambda_{0}+\delta_{0}\right) t} y(t)-e^{-\delta_{0} t} \frac{L\left(\lambda_{0} ; \phi\right)}{\beta_{\lambda_{0}}}-\frac{R\left(\lambda_{0}, \delta_{0} ; \phi\right)}{\eta_{\lambda_{0}, \delta_{0}}}-e^{\gamma_{0} t} \frac{K\left(\lambda_{0}, \delta_{0}, \gamma_{0} ; \phi\right)}{1+\xi_{\lambda_{0}, \delta_{0}, \gamma_{0}}}\right] \\
& \leq C_{2}\left(\lambda_{0}, \delta_{0}, \gamma_{0}, \gamma_{1} ; \phi\right)
\end{aligned}
$$

for all $t \geq 0$, where

$$
C_{1}\left(\lambda_{0}, \delta_{0}, \gamma_{0}, \gamma_{1} ; \phi\right)=\min _{-\tau \leq t \leq 0} e^{-\gamma_{1} t}\left[e^{-\left(\lambda_{0}+\delta_{0}\right) t} \phi(t)-e^{-\delta_{0} t} \frac{L\left(\lambda_{0} ; \phi\right)}{\beta_{\lambda_{0}}}-\frac{R\left(\lambda_{0}, \delta_{0} ; \phi\right)}{\eta_{\lambda_{0}, \delta_{0}}}-e^{\gamma_{0} t} \frac{K\left(\lambda_{0}, \delta_{0}, \gamma_{0} ; \phi\right)}{1+\xi_{\lambda_{0}, \delta_{0}, \gamma_{0}}}\right]
$$

and

$$
C_{2}\left(\lambda_{0}, \delta_{0}, \gamma_{0}, \gamma_{1} ; \phi\right)=\max _{-\tau \leq t \leq 0} e^{-\gamma_{1} t}\left[e^{-\left(\lambda_{0}+\delta_{0}\right) t} \phi(t)-e^{-\delta_{0} t} \frac{L\left(\lambda_{0} ; \phi\right)}{\beta_{\lambda_{0}}}-\frac{R\left(\lambda_{0}, \delta_{0} ; \phi\right)}{\eta_{\lambda_{0}, \delta_{0}}}-e^{\gamma_{0} t} \frac{K\left(\lambda_{0}, \delta_{0}, \gamma_{0} ; \phi\right)}{1+\xi_{\lambda_{0}, \delta_{0}, \gamma_{0}}}\right]
$$

We see immediately that inequalities (85) can equivalently be written as follows

$$
\begin{aligned}
C_{1}\left(\lambda_{0}, \delta_{0}, \gamma_{0}, \gamma_{1} ; \phi\right) e^{\left(\gamma_{1}-\gamma_{0}\right) t} & \leq e^{-\gamma_{0} t}\left[e^{-\left(\lambda_{0}+\delta_{0}\right) t} y(t)-e^{-\delta_{0} t} \frac{L\left(\lambda_{0} ; \phi\right)}{\beta_{\lambda_{0}}}-\frac{R\left(\lambda_{0}, \delta_{0} ; \phi\right)}{\eta_{\lambda_{0}, \delta_{0}}}\right]-\frac{K\left(\lambda_{0}, \delta_{0}, \gamma_{0} ; \phi\right)}{1+\xi_{\lambda_{0}, \delta_{0}, \gamma_{0}}} \\
& \leq C_{2}\left(\lambda_{0}, \delta_{0}, \gamma_{0}, \gamma_{1} ; \phi\right) e^{\left(\gamma_{1}-\gamma_{0}\right) t}, t \geq 0
\end{aligned}
$$

Hence, if $\gamma_{1}<\gamma_{0}$, then the solution $y$ of the IVP (1) and (2) satisfies (33). Also, we observe that (85) is equivalent to

$$
\begin{aligned}
& e^{\lambda_{0} t}\left[C_{1}\left(\lambda_{0}, \delta_{0}, \gamma_{0}, \gamma_{1} ; \phi\right) e^{\left(\delta_{0}+\gamma_{1}\right) t}+\frac{L\left(\lambda_{0} ; \phi\right)}{\beta_{\lambda_{0}}}+\frac{R\left(\lambda_{0}, \delta_{0} ; \phi\right)}{\eta_{\lambda_{0}, \delta_{0}}} e^{\delta_{0} t}+\frac{K\left(\lambda_{0}, \delta_{0}, \gamma_{0} ; \phi\right)}{1+\xi_{\lambda_{0}, \delta_{0}, \gamma_{0}}} e^{\left(\delta_{0}+\gamma_{0}\right) t}\right] \leq y(t) \\
& \leq e^{\lambda_{0} t}\left[C_{2}\left(\lambda_{0}, \delta_{0}, \gamma_{0}, \gamma_{1} ; \phi\right) e^{\left(\delta_{0}+\gamma_{1}\right) t}+\frac{L\left(\lambda_{0} ; \phi\right)}{\beta_{\lambda_{0}}}+\frac{R\left(\lambda_{0}, \delta_{0} ; \phi\right)}{\eta_{\lambda_{0}, \delta_{0}}} e^{\delta_{0} t}+\frac{K\left(\lambda_{0}, \delta_{0}, \gamma_{0} ; \phi\right)}{1+\xi_{\lambda_{0}, \delta_{0}, \gamma_{0}}} e^{\left(\delta_{0}+\gamma_{0}\right) t}\right]
\end{aligned}
$$

for all $t \geq 0$.

Proof. (Proof of Theorem 3.) Let $y$ be the solution of the IVP (1) and (2), and consider the function $z$ defined by (9). Consider also the functions $\Omega$ and $\Theta$ defined by (7) and (40), respectively. Note that, by Lemma 5.1, we necessarily have $1+\xi_{\lambda_{0}, \delta_{0}, \gamma_{0}} \neq 0$. As it has been shown in the proof of the Theorem 1, the fact that y satisfies (1) for $t \geq 0$ is equivalent to the fact that $\Theta$ satisfies (41) 2.12 for all $t \geq 0$.

Now we define

$$
h(t)=e^{\left(\gamma_{0}-\gamma_{1}\right) t} \Theta(t) \text { for } t \geq-\tau .
$$


Then we see that(41) holds for $t \geq 0$ if and only if $h$ satisfies

$$
\begin{aligned}
h(t)= & -e^{-\left(\lambda_{0}+\delta_{0}+\gamma_{0}\right) \tau} p_{2} \int_{-\tau}^{0} e^{\left(\gamma_{1}-\gamma_{0}\right) s} h(s+t) d s \\
& +e^{-\left(\lambda_{0}+\delta_{0}\right) \tau}\left(p_{2} \delta_{0}+q_{2}+2 p_{2} \lambda_{0}\right) \int_{0}^{\tau} e^{-\gamma_{0} s} \int_{-s}^{0} e^{\left(\gamma_{1}-\gamma_{0}\right) u} h(t+u) d u d s \\
& -e^{-\lambda_{0} \tau}\left(p_{2} \lambda_{0}^{2}+q_{2} \lambda_{0}+v_{2}\right) \int_{0}^{\tau} e^{-\delta_{0} s}\left[\int_{0}^{s} e^{-\gamma_{0} u} \int_{-u}^{0} e^{\left(\gamma_{1}-\gamma_{0}\right) w} h(t+w) d w d u\right] d s
\end{aligned}
$$

for all $t \geq 0$. By combining (51) and (89), we have

$$
h(t)=e^{-\gamma_{1} t}\left[e^{-\lambda_{0}+\delta_{0} t} y(t)-e^{-\delta_{0} t} \frac{L\left(\lambda_{0} ; \phi\right)}{\beta_{\lambda_{0}}}-\frac{R\left(\lambda_{0}, \delta_{0} ; \phi\right)}{\eta_{\lambda_{0}, \delta_{0}}}-e^{\gamma_{0} t} \frac{K\left(\lambda_{0}, \delta_{0}, \gamma_{0} ; \phi\right)}{1+\xi_{\lambda_{0}, \delta_{0}, \gamma_{0}}}\right]
$$

for $t \geq-\tau$. As solution $y$ satisfies the initial condition (2), we can use (91) as well as the definitions of $C_{1}\left(\lambda_{0}, \delta_{0}, \gamma_{0}, \gamma_{1} ; \phi\right)$ and $C_{2}\left(\lambda_{0}, \delta_{0}, \gamma_{0}, \gamma_{1} ; \phi\right)$ by (86) and (87), respectively, to see that

$$
C_{1}\left(\lambda_{0}, \delta_{0}, \gamma_{0}, \gamma_{1} ; \phi\right)=\min _{-\tau \leq t \leq 0} h(t) \quad \text { and } \quad C_{2}\left(\lambda_{0}, \delta_{0}, \gamma_{0}, \gamma_{1} ; \phi\right)=\max _{-\tau \leq t \leq 0} h(t)
$$

In view of (91) and (92), the double inequality (85) can equivalently written as follows

$$
\min _{-\tau \leq s \leq 0} h(s) \leq h(t) \leq \max _{-\tau \leq s \leq 0} h(s) \text { for all } \quad t \geq 0
$$

All we have to prove that (93) hold. We will use the fact that $h$ satisfies (90) for all $t \geq 0$ in order to show that (93) is valid. We restrict ourselves to proving that

$$
h(t) \geq \min _{-\tau \leq s \leq 0} h(s) \text { for every } \quad t \geq 0
$$

The proof of the inequality

$$
h(t) \leq \max _{-\tau \leq s \leq 0} h(s) \quad \text { for every } \quad t \geq 0
$$

can be obtained in a similar way, and so it is omitted. In the rest of the proof we will establish (94). In order to so, we consider an arbitrary real number $A$ with $A<\min _{-\tau \leq s \leq 0} h(s)$, i.e., with

$$
h(t)>A \text { for } \quad-\tau \leq t \leq 0
$$

We will show that

$$
h(t)>A \text { for all } t \geq 0
$$

To this end, let us assume that (96) fails to hold. Then, because of (95), there exists a point $t_{0}>0$ so that

$$
h(t)>A \text { for } \quad-\tau \leq t<t_{0}, \quad \text { and } \quad h\left(t_{0}\right)=A .
$$

Thus, by using (81) and (18), from (90) we obtain 


$$
\begin{aligned}
& A=h\left(t_{0}\right)=-e^{-\left(\lambda_{0}+\delta_{0}+\gamma_{0}\right) \tau} p_{2} \int_{-\tau}^{0} e^{\left(\gamma_{1}-\gamma_{0}\right) s} h\left(s+t_{0}\right) d s \\
& +e^{-\left(\lambda_{0}+\delta_{0}\right) \tau}\left(p_{2} \delta_{0}+q_{2}+2 p_{2} \lambda_{0}\right) \int_{0}^{\tau} e^{-\gamma_{0} s} \int_{-s}^{0} e^{\left(\gamma_{1}-\gamma_{0}\right) u} h\left(t_{0}+u\right) d u d s \\
& -e^{-\lambda_{0} \tau}\left(p_{2} \lambda_{0}^{2}+q_{2} \lambda_{0}+v_{2}\right) \int_{0}^{\tau} e^{-\delta_{0} s}\left[\int_{0}^{s} e^{-\gamma_{0} u} \int_{-u}^{0} e^{\left(\gamma_{1}-\gamma_{0}\right) w} h\left(t_{0}+w\right) d w d u\right] d s \\
& >A\left(-e^{-\left(\lambda_{0}+\delta_{0}+\gamma_{0}\right) \tau} p_{2} \int_{-\tau}^{0} e^{\left(\gamma_{1}-\gamma_{0}\right) s} d s+e^{-\left(\lambda_{0}+\delta_{0}\right) \tau}\left(p_{2} \delta_{0}+q_{2}+2 p_{2} \lambda_{0}\right) \int_{0}^{\tau} e^{-\gamma_{0} s} \int_{-s}^{0} e^{\left(\gamma_{1}-\gamma_{0}\right) u} d u d s\right. \\
& \left.-e^{-\lambda_{0} \tau}\left(p_{2} \lambda_{0}^{2}+q_{2} \lambda_{0}+v_{2}\right) \int_{0}^{\tau} e^{-\delta_{0} s}\left[\int_{0}^{s} e^{-\gamma_{0} u} \int_{-u}^{0} e^{\left(\gamma_{1}-\gamma_{0}\right) w} d w d u\right] d s\right) \\
& =\frac{A}{\gamma_{1}-\gamma_{0}}\left(-e^{-\left(\lambda_{0}+\delta_{0}+\gamma_{0}\right) \tau} p_{2}\left[1-e^{-\left(\gamma_{1}-\gamma_{0}\right) \tau}\right]+e^{-\left(\lambda_{0}+\delta_{0}\right) \tau}\left(p_{2} \delta_{0}+q_{2}+2 p_{2} \lambda_{0}\right) \int_{0}^{\tau}\left[e^{-\gamma_{0} s}-e^{-\gamma_{1} s}\right] d s\right. \\
& \left.-e^{-\lambda_{0} \tau}\left(p_{2} \lambda_{0}^{2}+q_{2} \lambda_{0}+v_{2}\right) \int_{0}^{\tau} e^{-\delta_{0} s}\left[\int_{0}^{s}\left[e^{-\gamma_{0} u}-e^{-\gamma_{1} u}\right] d u\right] d s\right) \\
& =\frac{A}{\gamma_{1}-\gamma_{0}}\left(p_{2}\left[e^{-\left(\lambda_{0}+\delta_{0}+\gamma_{1}\right) \tau}-e^{-\left(\lambda_{0}+\delta_{0}+\gamma_{0}\right) \tau}\right]\right. \\
& +e^{-\left(\lambda_{0}+\delta_{0}\right) \tau}\left(p_{2} \delta_{0}+q_{2}+2 p_{2} \lambda_{0}\right)\left[\gamma_{1}^{-1}\left(e^{-\gamma_{1} \tau}-1\right)-\gamma_{0}^{-1}\left(e^{-\gamma_{0} \tau}-1\right)\right] \\
& -e^{-\lambda_{0} \tau}\left(p_{2} \lambda_{0}^{2}+q_{2} \lambda_{0}+v_{2}\right) \gamma_{1}^{-1}\left[\left(\gamma_{1}+\delta_{0}\right)^{-1}\left(1-e^{-\left(\gamma_{1}+\ddot{a}_{0}\right) \tau}\right)-\delta_{0}^{-1}\left(1-e^{-\delta_{0} \tau}\right)\right] \\
& \left.\left.-\gamma_{0}^{-1}\left[\left(\gamma_{0}+\delta_{0}\right)^{-1}\left(1-e^{-\left(\gamma_{0}+\delta_{0}\right) \tau}\right)-\delta_{0}^{-1}\left(1-e^{-\delta_{0} \tau}\right)\right]\right\}\right) \\
& =\frac{A}{\gamma_{1}-\gamma_{0}}\left(-p_{2} e^{-\left(\lambda_{0}+\delta_{0}+\gamma_{0}\right) \tau}+e^{-\left(\lambda_{0}+\delta_{0}\right) \tau}\left(p_{2} \delta_{0}+q_{2}+2 p_{2} \lambda_{0}\right) \gamma_{0}^{-1}\left(1-e^{-\gamma_{0} \tau}\right)\right. \\
& -e^{-\lambda_{0} \tau}\left(p_{2} \lambda_{0}^{2}+q_{2} \lambda_{0}+v_{2}\right) \gamma_{0}^{-1}\left[\delta_{0}^{-1}\left(1-e^{-\delta_{0} \tau}\right)-\left(\gamma_{0}+\delta_{0}\right)^{-1}\left(1-e^{-\left(\gamma_{0}+\delta_{0}\right) \tau}\right)\right] \\
& +p_{2} e^{-\left(\lambda_{0}+\delta_{0}+\gamma_{1}\right) \tau}-e^{-\left(\lambda_{0}+\delta_{0}\right) \tau}\left(p_{2} \delta_{0}+q_{2}+2 p_{2} \lambda_{0}\right) \gamma_{1}^{-1}\left(1-e^{-\gamma_{1} \tau}\right) \\
& \left.+e^{-\lambda_{0} \tau}\left(p_{2} \lambda_{0}^{2}+q_{2} \lambda_{0}+v_{2}\right) \gamma_{1}^{-1}\left[\delta_{0}^{-1}\left(1-e^{-\delta_{0} \tau}\right)-\left(\gamma_{1}+\delta_{0}\right)^{-1}\left(1-e^{-\left(\gamma_{1}+\delta_{0}\right) \tau}\right)\right]\right) \\
& =\frac{A}{\gamma_{1}-\gamma_{0}}\left(p_{1}-3 \lambda_{0}-2 \delta_{0}-\gamma_{0}+\gamma_{1}-p_{1}+3 \lambda_{0}+2 \delta_{0}\right)=A \text {. }
\end{aligned}
$$

We have thus arrived at a contradiction and so (96) is true. Since (96) is satisfied for all real numbers $A$ with $A<\min _{-\tau \leq s \leq 0} h(s)$, it follows that (94) is always fulfilled. The proof of the theorem is complete.

\section{Sufficient conditions for the characteristic equation to have a real root with the property required}

In this section, we give some conditions, under which the characteristic equation (18) has a real root $\gamma_{0}$ with the property (32).

Lemma 2. Let $\lambda_{0}$ and $\delta_{0}$ be real root of the characteristic equations (3) and (5), respectively. Assume that

$$
\begin{array}{r}
-p_{2} e^{-\left(p_{1}-2 \lambda_{0}-\delta_{0}-\frac{1}{\tau}\right) \tau}+e^{-\left(\lambda_{0}+\delta_{0}\right) \tau}\left(p_{2} \delta_{0}+q_{2}+2 p_{2} \lambda_{0}\right) \int_{0}^{\tau} e^{-\left(p_{1}-3 \lambda_{0}-2 \delta_{0}-\frac{1}{\tau}\right) s} d s \\
-e^{-\lambda_{0} \tau}\left(p_{2} \lambda_{0}^{2}+q_{2} \lambda_{0}+v_{2}\right) \int_{0}^{\tau} e^{-\delta_{0} s}\left(\int_{0}^{s} e^{-\left(p_{1}-3 \lambda_{0}-2 \delta_{0}-\frac{1}{\tau}\right) u} d u\right) d s<\frac{1}{\tau}
\end{array}
$$


and

$$
\begin{array}{r}
\left|p_{2}\right| \tau e^{-\left(p_{1}-2 \lambda_{0}-\delta_{0}-\frac{1}{\tau}\right) \tau}+e^{-\left(\lambda_{0}+\delta_{0}\right) \tau}\left|p_{2} \delta_{0}+q_{2}+2 p_{2} \lambda_{0}\right| \int_{0}^{\tau} s e^{-\left(p_{1}-3 \lambda_{0}-2 \delta_{0}-\frac{1}{\tau}\right) s} d s \\
+e^{-\lambda_{0} \tau}\left|p_{2} \lambda_{0}^{2}+q_{2} \lambda_{0}+v_{2}\right| \int_{0}^{\tau} e^{-\delta_{0} s}\left(\int_{0}^{s} u e^{-\left(p_{1}-3 \lambda_{0}-2 \delta_{0}-\frac{1}{\tau}\right) u} d u\right) d s \leq 1
\end{array}
$$

Then, in the interval $\left(p_{1}-3 \lambda_{0}-2 \delta_{0}-\frac{1}{\tau}, \infty\right)$, the characteristic equation (18) has a unique root $\gamma_{0}$; this root satisfies (32), and the root $\gamma_{0}$ is less than $p_{1}-3 \lambda_{0}-2 \delta_{0}+\frac{1}{\tau}$, provided that

$$
\begin{gathered}
-p_{2} e^{-\left(p_{1}-2 \lambda_{0}-\delta_{0}+\frac{1}{\tau}\right) \tau}+e^{-\left(\lambda_{0}+\delta_{0}\right) \tau}\left(p_{2} \delta_{0}+q_{2}+2 p_{2} \lambda_{0}\right) \int_{0}^{\hat{o}} e^{-\left(p_{1}-3 \lambda_{0}-2 \delta_{0}+\frac{1}{\tau}\right) s} d s \\
-e^{-\lambda_{0} \tau}\left(p_{2} \lambda_{0}^{2}+q_{2} \lambda_{0}+v_{2}\right) \int_{0}^{\tau} e^{-\delta_{0} s}\left(\int_{0}^{s} e^{-\left(p_{1}-3 \lambda_{0}-2 \delta_{0}+\frac{1}{\tau}\right) u} d u\right) d s>-\frac{1}{\tau} .
\end{gathered}
$$

Proof. Consider the real-valued function $G$ defined by (82). The derivative $G^{\prime}$ of $G$ is given by (83). It follows from (82) that

$$
\begin{aligned}
G\left(p_{1}-3 \lambda_{0}-2 \delta_{0}-\frac{1}{\tau}\right)= & \left(p_{1}-3 \lambda_{0}-2 \delta_{0}-\frac{1}{\tau}\right)-\left(p_{1}-3 \lambda_{0}-2 \delta_{0}\right) \\
& -p_{2} e^{-\left(p_{1}-2 \lambda_{0}-\delta_{0}-\frac{1}{\tau}\right) \tau}+e^{-\left(\lambda_{0}+\delta_{0}\right) \tau}\left(p_{2} \delta_{0}+q_{2}+2 p_{2} \lambda_{0}\right) \int_{0}^{\tau} e^{-\left(p_{1}-3 \lambda_{0}-2 \delta_{0}-\frac{1}{\tau}\right) s} d s \\
& -e^{-\lambda_{0} \tau}\left(p_{2} \lambda_{0}^{2}+q_{2} \lambda_{0}+v_{2}\right) \int_{0}^{\tau} e^{-\delta_{0} s}\left(\int_{0}^{s} e^{-\left(p_{1}-3 \lambda_{0}-2 \delta_{0}-\frac{1}{\tau}\right) u} d u\right) d s \\
= & -\frac{1}{\tau}-p_{2} e^{-\left(p_{1}-2 \lambda_{0}-\delta_{0}-\frac{1}{\tau}\right) \tau}+e^{-\left(\lambda_{0}+\delta_{0}\right) \tau}\left(p_{2} \delta_{0}+q_{2}+2 p_{2} \lambda_{0}\right) \int_{0}^{\tau} e^{-\left(p_{1}-3 \lambda_{0}-2 \delta_{0}-\frac{1}{\tau}\right) s} d s \\
& -e^{-\lambda_{0} \tau}\left(p_{2} \lambda_{0}^{2}+q_{2} \lambda_{0}+v_{2}\right) \int_{0}^{\tau} e^{-\delta_{0} s}\left(\int_{0}^{s} e^{-\left(p_{1}-3 \lambda_{0}-2 \delta_{0}-\frac{1}{\tau}\right) u} d u\right) d s \\
\leq & -\frac{1}{\tau}+\frac{1}{\tau}=0
\end{aligned}
$$

and consequently, by (97), it holds

$$
G\left(p_{1}-3 \lambda_{0}-2 \delta_{0}-\frac{1}{\tau}\right)<0
$$

Moreover, from (82) we obtain, for $\gamma \geq p_{1}-3 \lambda_{0}-2 \delta_{0}-\frac{1}{\tau}$,

$$
\begin{aligned}
G(\gamma) \geq & \gamma-\left(p_{1}-3 \lambda_{0}-2 \delta_{0}\right)-\left|p_{2}\right| e^{-\left(\lambda_{0}+\delta_{0}+\gamma\right) \tau}-e^{-\left(\lambda_{0}+\delta_{0}\right) \tau}\left|p_{2} \delta_{0}+q_{2}+2 p_{2} \lambda_{0}\right| \int_{0}^{\tau} e^{-\gamma s} d s \\
& -e^{-\lambda_{0} \tau}\left|p_{2} \lambda_{0}^{2}+q_{2} \lambda_{0}+v_{2}\right| \int_{0}^{\tau} e^{-\delta_{0} s}\left(\int_{0}^{s} e^{-\gamma u} d u\right) d s \\
\geq & \gamma-\left(p_{1}-3 \lambda_{0}-2 \delta_{0}\right)-\left|p_{2}\right| e^{-\left(p_{1}-2 \lambda_{0}-\delta_{0}-\frac{1}{\tau}\right) \tau}-e^{-\left(\lambda_{0}+\delta_{0}\right) \tau}\left|p_{2} \delta_{0}+q_{2}+2 p_{2} \lambda_{0}\right| \int_{0}^{\tau} e^{-\left(p_{1}-3 \lambda_{0}-2 \delta_{0}-\frac{1}{\tau}\right) s} d s \\
& -e^{-\lambda_{0} \tau}\left|p_{2} \lambda_{0}^{2}+q_{2} \lambda_{0}+v_{2}\right| \int_{0}^{\tau} e^{-\delta_{0} s}\left(\int_{0}^{s} e^{-\left(p_{1}-3 \lambda_{0}-2 \delta_{0}-\frac{1}{\tau}\right) u} d u\right) d s .
\end{aligned}
$$

Therefore,

$$
G(\infty)=\infty
$$


Furthermore, using (83), we have, for every $\gamma>p_{1}-3 \lambda_{0}-2 \delta_{0}-\frac{1}{\tau}$,

$$
\begin{aligned}
G^{\prime}(\gamma) \geq & 1-\left|p_{2}\right| \tau e^{-\left(\lambda_{0}+\delta_{0}+\gamma\right) \tau}-e^{-\left(\lambda_{0}+\delta_{0}\right) \tau}\left|p_{2} \delta_{0}+q_{2}+2 p_{2} \lambda_{0}\right| \int_{0}^{\tau} s e^{-\gamma_{s}} d s \\
& -e^{-\lambda_{0} \tau}\left|p_{2} \lambda_{0}^{2}+q_{2} \lambda_{0}+v_{2}\right| \int_{0}^{\tau} e^{-\delta_{0} s}\left(\int_{0}^{s} u e^{-\gamma u} d u\right) d s \\
\geq & 1-\left|p_{2}\right| \tau e^{-\left(p_{1}-2 \lambda_{0}-\delta_{0}-\frac{1}{\tau}\right) \tau}-e^{-\left(\lambda_{0}+\delta_{0}\right) \tau}\left|p_{2} \delta_{0}+q_{2}+2 p_{2} \lambda_{0}\right| \int_{0}^{\tau} s e^{-\left(p_{1}-3 \lambda_{0}-2 \delta_{0}-\frac{1}{\tau}\right) s} d s \\
& -e^{-\lambda_{0} \tau}\left|p_{2} \lambda_{0}^{2}+q_{2} \ddot{\mathrm{e}}_{0}+v_{2}\right| \int_{0}^{\tau} e^{-\delta_{0} s}\left(\int_{0}^{s} u e^{-\left(p_{1}-3 \lambda_{0}-2 \delta_{0}-\frac{1}{\tau}\right) u} d u\right) d s \\
\geq & 1-1=0 .
\end{aligned}
$$

Consequently, in view of (98), it holds $G^{\prime}(\gamma)>0$ for all $\gamma>p_{1}-3 \lambda_{0}-2 \delta_{0}-\frac{1}{\tau}$, which implies that $G$ is strictly increasing on $\left(p_{1}-3 \lambda_{0}-2 \delta_{0}-\frac{1}{\tau}, \infty\right)$. By using this fact as well as (100) and (101), we conclude that, in the intreval $\left(p_{1}-3 \lambda_{0}-2 \delta_{0}-\frac{1}{\tau}, \infty\right)$, the equation $G(\gamma)=0$ (which coincides with (18)) has a unique real root $\gamma_{0}$. This root satisfies (32). Indeed, by using again (98), we have

$$
\begin{gathered}
\mu_{\lambda_{0}, \delta_{0}, \gamma_{0}}<\left|p_{2}\right| \tau e^{-\left(p_{1}-2 \lambda_{0}-\delta_{0}-\frac{1}{\tau}\right) \tau}+e^{-\left(\lambda_{0}+\delta_{0}\right) \tau}\left|p_{2} \delta_{0}+q_{2}+2 p_{2} \lambda_{0}\right| \int_{0}^{\tau} s e^{-\left(p_{1}-3 \lambda_{0}-2 \delta_{0}-\frac{1}{\tau}\right) s} d s \\
+e^{-\lambda_{0} \tau}\left|p_{2} \lambda_{0}^{2}+q_{2} \lambda_{0}+v_{2}\right| \int_{0}^{\tau} e^{-\delta_{0} s}\left(\int_{0}^{s} u e^{-\left(p_{1}-3 \lambda_{0}-2 \delta_{0}-\frac{1}{\tau}\right) u} d u\right) d s \leq 1 .
\end{gathered}
$$

Finally, let us assume that (99) holds. Then it follows from (82) that

$$
\begin{aligned}
G\left(p_{1}-3 \lambda_{0}-2 \delta_{0}+\frac{1}{\tau}\right)= & \frac{1}{\tau}-p_{2} e^{-\left(p_{1}-2 \lambda_{0}-\delta_{0}+\frac{1}{\tau}\right) \tau}+e^{-\left(\lambda_{0}+\delta_{0}\right) \tau}\left(p_{2} \delta_{0}+q_{2}+2 p_{2} \lambda_{0}\right) \int_{0}^{\tau} e^{-\left(p_{1}-3 \lambda_{0}-2 \delta_{0}+\frac{1}{\tau}\right) s} d s \\
& -e^{-\lambda_{0} \tau}\left(p_{2} \lambda_{0}^{2}+q_{2} \lambda_{0}+v_{2}\right) \int_{0}^{\tau} e^{-\delta_{0} s}\left(\int_{0}^{s} e^{-\left(p_{1}-3 \lambda_{0}-2 \delta_{0}+\frac{1}{\tau}\right) u} d u\right) d s \\
> & \frac{1}{\tau}-\frac{1}{\tau}=0 .
\end{aligned}
$$

As $G\left(p_{1}-3 \lambda_{0}-2 \delta_{0}+\frac{1}{\tau}\right)>0$, we see that $\gamma_{0}$ must be less than $p_{1}-3 \lambda_{0}-2 \delta_{0}+\frac{1}{\tau}$. This completes the proof of the lemma.

Lemma 3. Suppose that statement (81) is true. Let $\lambda_{0}$ and $\delta_{0}$ be real root of the characteristic equations (3) and (5), respectively. Then we have

(a) In the interval $\left[p_{1}-3 \lambda_{0}-2 \delta_{0}, \infty\right)$, the characteristic equation (18) has no roots.

(b) Assume that (97) holds. Then (i) $\gamma=p_{1}-3 \lambda_{0}-2 \delta_{0}-\frac{1}{\tau}$ is not a root of the characteristic equation (18). (ii) In the interval $\left(p_{1}-3 \lambda_{0}-2 \delta_{0}-\frac{1}{\tau}, p_{1}-3 \lambda_{0}-2 \delta_{0}\right)$, (18) has a unique root. (iii) In the interval $\left(-\infty, p_{1}-3 \lambda_{0}-2 \delta_{0}-\frac{1}{\tau}\right)$, (18) has a unique root.

\section{Proof.}

(a) Let $\widehat{\gamma}$ be real root of the characteristic equation. Using (18), we can immediately see that

$$
\begin{aligned}
& p_{2} e^{-\left(\lambda_{0}+\delta_{0}+\widehat{\gamma}\right) \tau}-e^{-\left(\lambda_{0}+\delta_{0}\right) \tau}\left(p_{2} \delta_{0}+q_{2}+2 p_{2} \lambda_{0}\right) \int_{0}^{\tau} e^{-\widehat{\gamma} s} d s \\
& +e^{-\lambda_{0} \tau}\left(p_{2} \lambda_{0}^{2}+q_{2} \lambda_{0}+v_{2}\right) \int_{0}^{\tau} e^{-\delta_{0} s}\left(\int_{0}^{s} e^{-\widehat{\gamma} u} d u\right) d s<0
\end{aligned}
$$


Hence, from (18) it follows that $\widehat{\gamma}-\left(p_{1}-3 \lambda_{0}-2 \delta_{0}\right)<0$, i.e., $\widehat{\gamma}<\left(p_{1}-3 \lambda_{0}-2 \delta_{0}\right)$. We have thus proved that every real root of (18) is always less than $p_{1}-3 \lambda_{0}-2 \delta_{0}$.

(b) Consider the real-valued function $G$ defined by (82). As in the proof of Lemma 1, we see that (84) holds and consequently

$$
G \text { is convex on } \mathbb{R} \text {. }
$$

Next, we observe that, as in the proof of Lemma 2, assumption (97) means that (100) holds true. Inequality (100) implies, in particular, that $\gamma=p_{1}-3 \lambda_{0}-2 \delta_{0}-\frac{1}{\tau}$ is not a root of the characteristic equation (18). From (82) we obtain

$$
\begin{aligned}
G\left(p_{1}-3 \lambda_{0}-2 \delta_{0}\right)= & -p_{2} e^{-\left(p_{1}-2 \lambda_{0}-\delta_{0}\right) \tau}+e^{-\left(\lambda_{0}+\delta_{0}\right) \tau}\left(p_{2} \delta_{0}+q_{2}+2 p_{2} \lambda_{0}\right) \int_{0}^{\tau} e^{-\left(p_{1}-3 \lambda_{0}-2 \delta_{0}\right) s} d s \\
& -e^{-\lambda_{0} \tau}\left(p_{2} \lambda_{0}^{2}+q_{2} \lambda_{0}+v_{2}\right) \int_{0}^{\tau} e^{-\delta_{0} s}\left(\int_{0}^{s} e^{-\left(p_{1}-3 \lambda_{0}-2 \delta_{0}\right) u} d u\right) d s
\end{aligned}
$$

So, by using (81), we conclude that

$$
G\left(p_{1}-3 \lambda_{0}-2 \delta_{0}\right)>0
$$

Furthermore, from (82) we get $G(\gamma) \geq \gamma-\left(p_{1}-3 \lambda_{0}-2 \delta_{0}\right)-p_{2} e^{-\left(\lambda_{0}+\delta_{0}+\gamma\right) \tau}$ for $\gamma \in \mathbb{R}$. Using this inequality, it is not difficult to show that

$$
G(-\infty)=\infty
$$

From (100), (102) and (103) it follows that, in the interval $\left(p_{1}-3 \lambda_{0}-2 \delta_{0}-\frac{1}{\tau}, p_{1}-3 \lambda_{0}-2 \delta_{0}\right)$, the characteristic equation (18) has a unique root. Moreover, (100), (102) and (104) guarantee that, in the interval $\left(-\infty, p_{1}-3 \lambda_{0}-2 \delta_{0}-\frac{1}{\tau}\right),(18)$ has also a unique root. The proof of the lemma is complete.

\section{Competing interests}

The authors declare that they have no competing interests.

\section{Authors' contributions}

All authors have contributed to all parts of the article. All authors read and approved the final manuscript.

\section{References}

[1] R. Bellman, and K. Cooke, Differential-Difference Equations. Academic Press, New York, (1963).

[2] B. Cahlon and D. Schmidt, Stability criteria for certain third-order delay differential equations, Journal of Computational and Applied Mathematics, 188 (2006) 319-335.

[3] B. Cahlon, D. Schmidt, Asymptotic stability of amechanical robotics model with damping and delay, Journal of Mathematical Analysis and Applications 303 (2005) 36-53.

[4] R.D. Driver, Ordinary and Delay Differential Equations, Springer-Verlag, New York, 1977.

[5] L.E. El'sgol'ts and S.B. Norkin, Introduction to the Theory and Application of Differential Equations with Deviating Arguments, Academic Pres, New York, London, 1973. 
[6] J.K. Hale and S.M. Verduyn Lunel, Introduction to Functional Differential Equations, Springer, Berlin, Heidelberg, New York, 1993.

[7] V. Kolmanovski and A. Myshkis, Applied Theory of Functional Differential Equations, Kluver Academic, Dordrecht, 1992.

[8] V. Lakshmikantham, L. Wen, and B. Zhang, Theory of Differential Equations with Unbounded Delay, Kluwer Academic Publishers, London, 1994.

[9] Ch.G. Philos and I.K. Purnaras, Periodic first order linear neutral delay differential equations, Applied Mathematics and Computation, 117 (2001) 203-222.

[10] Ch.G. Philos and I.K. Purnaras, Asymptotic properties, nonoscillation, and stability for scalar first order linear autonomous neutral delay differential equations, Electronic Journal of Differential Equations, Vol. 2004 (2004), No. 03, pp. 1-17.

[11] Ch.G. Philos and I.K. Purnaras, Behavior of the solutions to second order linear autonomous delay differential equations, Electronic Journal of Differential Equations, Vol. 2007 (2007), No. 106, pp. 1-35.

[12] A.F. Yeniçerioğlu, The behavior of solutions of second order delay differential equations, Journal of Mathematical Analysis and Applications, 332 (2007) 1278-1290. 